\title{
SHOOTING METHOD FOR NONLINEAR SINGULARLY PERTURBED BOUNDARY-VALUE PROBLEMS
}

\author{
by
}

\author{
C. H. Ou* and R. Wong*
}

\begin{abstract}
Asymptotic formulas, as $\varepsilon \rightarrow 0^{+}$, are derived for the solutions of the nonlinear differential equation $\varepsilon u^{\prime \prime}+Q(u)=0$ with boundary conditions $u(-1)=u(1)=0$ or $u^{\prime}(-1)=$ $u^{\prime}(1)=0$. The nonlinear term $Q(u)$ behaves like a cubic; it vanishes at $s_{-}, 0, s_{+}$and nowhere else in $\left[s_{-}, s_{+}\right]$, where $s_{-}<0<s_{+}$. Furthermore, $Q^{\prime}\left(s_{ \pm}\right)<0, Q^{\prime}(0)>0$ and the integral of $Q$ on the interval $\left[s_{-}, s_{+}\right]$is zero. Solutions to these boundary-value problems are shown to exhibit internal shock layers, and the error terms in the asymptotic approximations are demonstrated to be exponentially small. Estimates are obtained for the number of internal shocks that a solution can have, and the total numbers of solutions to these problems are also given. All results here are established rigorously in the mathematical sense.
\end{abstract}

* Department of Mathematics, City University of Hong Kong, Tat Chee Avenue, Kowloon, Hong Kong. 


\section{INTRODUCTION}

In this paper, we consider the singularly perturbed two-point problem

$$
\varepsilon u^{\prime \prime}+Q(u)=0, \quad-1<x<1,
$$

with boundary conditions

$$
u(-1)=u(1)=0
$$

or

$$
u^{\prime}(-1)=u^{\prime}(1)=0,
$$

where $\varepsilon$ is a small positive parameter. Throughout the paper, we shall assume that the nonlinear term $Q(u)$ vanishes at $s_{-}, 0, s_{+}$and nowhere else in $\left[s_{-}, s_{+}\right]$, where $s_{-}<0<s_{+}$. Furthermore, we assume that $Q^{\prime}\left(s_{ \pm}\right)<0, Q^{\prime}(0)>0$ and

$$
\int_{s_{-}}^{s_{+}} Q(s) d s=0 .
$$

The graph of the function $Q(u)$ has the typical shape shown in Figure 1.

Equation (1.1) can be considered as the equation of motion of a nonlinear spring with spring constant large compared to the mass. It is also the equilibrium equation associated with the Ginzburg-Landau model

$$
u_{t}=\varepsilon u_{x x}+Q(u), \quad-1<x<1, \quad t \geq 0,
$$

with various boundary conditions at $x= \pm 1$. In (1.5), $Q(u)=-\widetilde{V}^{\prime}(u)$, where $\widetilde{V}(u)$ is a double well potential with wells of equal depth located at the preferred phases $u=s_{-}$and $u=s_{+}$.

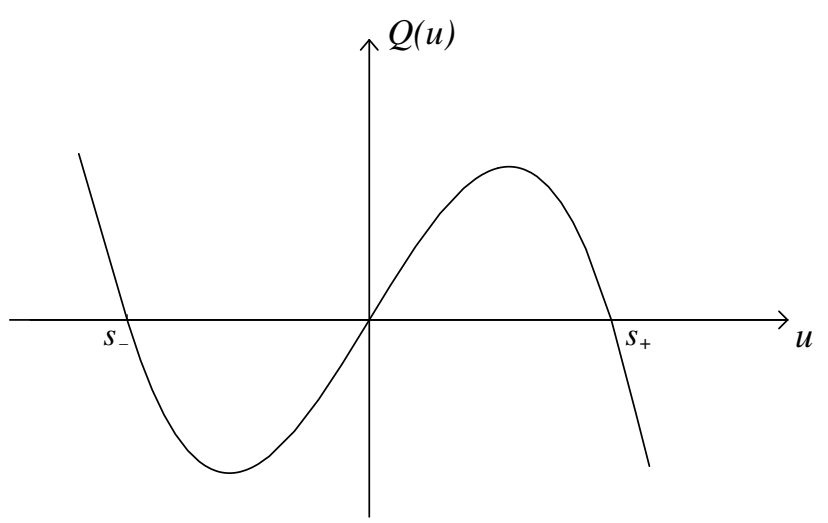

Figure 1. Graph of $Q(u)$.

The problem of finding asymptotic behavior of the solutions to (1.1) \& (1.2) or (1.1) \& (1.3) has been studied earlier by O'Malley [5], using a phase-plane analysis. Although 
his approach provides useful qualitative information about the solutions with internal layer behavior, it does not give quantitative information such as asymptotic formulas for the solutions. The best known approach to derive such formulas is probably the method of matched asymptotics. But, as was shown by Carrier and Pearson [1, p.202], a routine application of this method will not lead to the determination of the locations of the internal layers, thus creating spurious solutions. To overcome this difficulty, Lange [3] extended the method of matched asymptotics by including exponentially small terms in the expansion of the solution; see also MacGillivray [4]. There are two difficulties with Lange's approach; namely, (i) explicit expressions for the internal layer solutions must be known a priorily, (ii) second-order terms in the asymptotic expansions are needed to determine the layer positions of the leading order approximate solutions. An alternative approach has been introduced by Ward [8], which he later called the projection method (see [7, p.496]). Ward's method is an extension of the variational approach adopted by Kath et al [2], and does not require the knowledge of the explicit form of the internal layer solution. However, as stated by himself in [8, p.98], he is not able to determine the number of solutions to (1.1) for small fixed $\varepsilon$. More recently, Reyna and Ward [7] introduced another method, which involves a nonlinear WKB-type transformation for (1.1). The advantage of this method is that it avoids the use of exponential asymptotics, i.e., it is sufficient to use just the conventional singular perturbation approach on the transformed problem.

Despite the usefulness of all these methods mentioned above in providing approximate solutions to the problem (1.1) \& (1.2) or (1.1) \& (1.3) they have a common defect from a mathematical point of view; that is, none of the arguments used in these methods can be modified to show that for each approximate solution, there is one and only one true solution, and that their difference in absolute value tends to zero as $\varepsilon$ approaches zero. A first attempt in this direction was made in [6], where only the special case $Q(u)=1-u^{2}$ was considered. For instance, it was shown in [6] that in this special case, there exists exactly one solution $u_{1}(x, \varepsilon)$ to $(1.1) \&(1.2)$ satisfying

$$
u_{1}(x, \varepsilon)=\widetilde{u}_{1}(x, \varepsilon)+q\left(\frac{x}{\sqrt{\varepsilon}}\right)+O\left(e^{-\sqrt{2 / \varepsilon}}\right),
$$

where

$$
\begin{aligned}
\widetilde{u}_{1}(x, \varepsilon)=-1 & +3 \operatorname{sech}^{2}\left(\frac{x+1}{\sqrt{2 \varepsilon}}+\ln (\sqrt{3}+\sqrt{2})\right) \\
+ & 3 \operatorname{sech}^{2}\left(\frac{1-x}{\sqrt{2 \varepsilon}}+\ln (\sqrt{3}+\sqrt{2})\right)
\end{aligned}
$$

and

$$
q(\zeta)=\frac{12 e^{\sqrt{2} \zeta}}{\left(1+e^{\sqrt{2} \zeta}\right)^{2}} .
$$

The graph of the solution $u_{1}(x, \varepsilon)$ is shown in Figure 2 . 


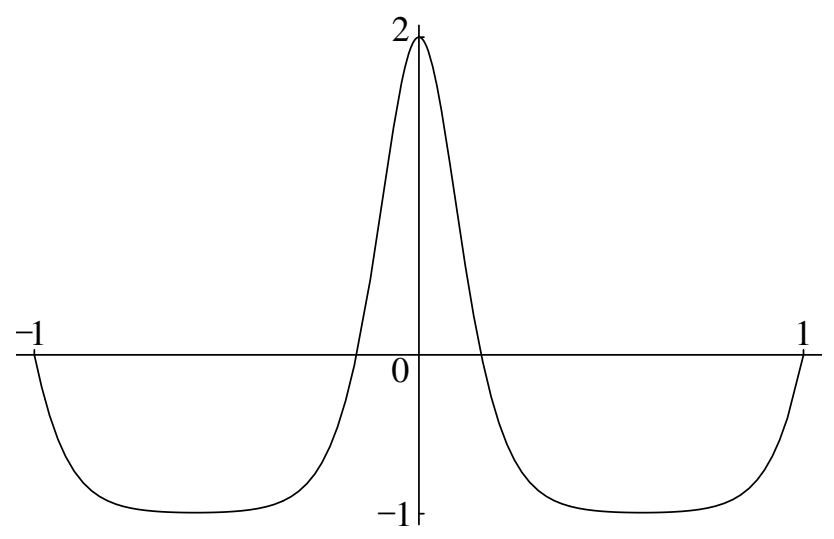

Figure 2. Graph of the solution $u_{1}(x, \varepsilon)$ when $\varepsilon=0.01$

The internal layer near the origin is called a spike. Furthermore, it was shown in [6] that if $n(\varepsilon)$ denotes the number of solutions to the boundary-value problem (BVP) - (1.1) $\&(1.2)$ when $Q(u)=1-u^{2}$, then we have the asymptotic formula

$$
n(\varepsilon) \sim \frac{1.64}{\sqrt{\varepsilon}} \quad \text { as } \varepsilon \rightarrow 0^{+} .
$$

As to the maximum number, say $N(\varepsilon)$, of spikes that a solution to $(1.1) \&(1.2)$ can have, we have the estimate

$$
N(\varepsilon) \leq \frac{0.41}{\sqrt{\varepsilon}}+1
$$

In the present paper, we shall establish corresponding results for the BVP (1.1) \& (1.2) or (1.1) \& (1.3), when $Q(u)$ is of the form shown in Figure 1. For instance, in the case when $Q(u)=2 u-2 u^{3}$ which was considered by Lange [3], we will show that there exist exactly two solutions $u_{1,1}(x, \varepsilon)$ and $u_{1,2}(x, \varepsilon)$ to $(1.1) \&(1.2)$ such that

$$
u_{1,2}(x, \varepsilon)=u_{1,1}(-x, \varepsilon)
$$

and

$$
\begin{aligned}
u_{1,1}(x, \varepsilon)= & \tanh \left(\frac{x+1}{\sqrt{\varepsilon}}\right)+\tanh \left(-\frac{x}{\sqrt{\varepsilon}}\right) \\
& +\tanh \left(\frac{x-1}{\sqrt{\varepsilon}}\right)+O\left(e^{-1 / \sqrt{\varepsilon}}\right) .
\end{aligned}
$$

The graph of $u_{1,1}(x, \varepsilon)$ is shown in Figure 3. The internal layer near $x=0$ in this case is called a shock. An estimate for the maximum number $n_{1}(\varepsilon)$ of shocks is given by 


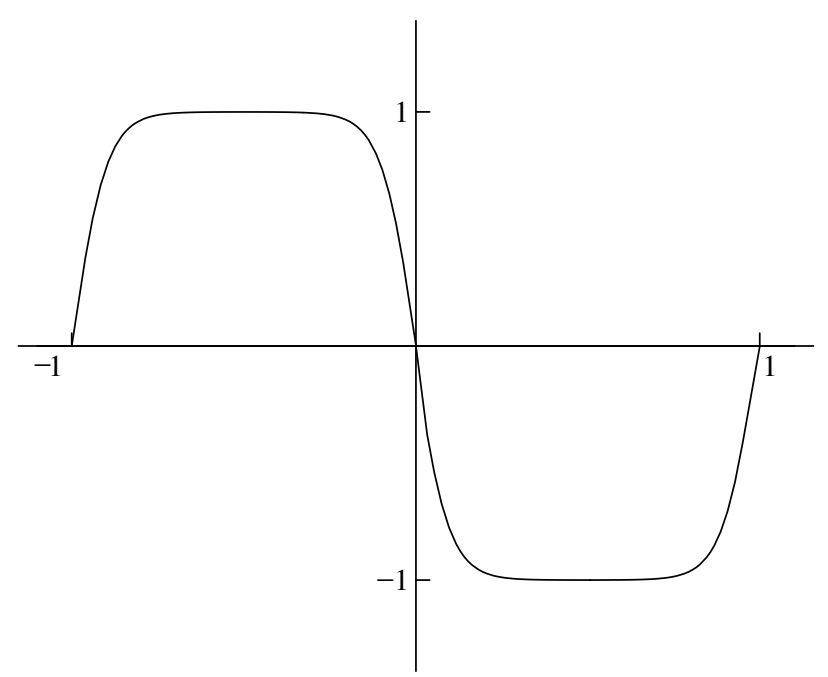

Figure 3. Graph of $u_{1,1}(x, \varepsilon)$ when $\varepsilon=0.01$.

$$
n_{1}(\varepsilon) \leq \frac{2}{\pi} \sqrt{\frac{2}{\varepsilon}}-1
$$

and the number $n_{2}(\varepsilon)$ of solutions to $(1.1)-(1.2)$ behaves like

$$
n_{2}(\varepsilon) \sim \frac{4}{\pi} \sqrt{\frac{2}{\varepsilon}}
$$

The presentation of this paper is arranged as follows. In $\S 2$, we consider an initialvalue problem (IVP), which is directly related to the BVP (1.1) - (1.2). We show that the existence of a solution to (1.1) - (1.2) depends very much on the slope of the solution to this IVP at $x=-1$. We also establish some properties about the lengths of the intervals in which the solution to this IVP is above or below the $x$-axis. In addition, we give estimates for the number of solutions to (1.1) - (1.2), and the maximum number of shocks that a solution can have. In $\S 3$, we examine the asymptotic nature of the approximate solutions in the case when there is no shocks or at most one shock. In fact, we shall prove that the differences between approximate solutions and true solutions are exponentially small. The results for the general case with $n$ shocks are presented in $\S 4$. In $\S 5$, we state the corresponding results for the BVP (1.1) \& (1.3). Two special but typical examples are given in $\S 6$. The final section contains discussions of some cases not touched upon in the previous sections, including the boundary-value problem consisting of equation (1.1) and the boundary conditions

$$
\sqrt{\varepsilon} u^{\prime}(1)+k_{r}\left(u(1)-s_{+}\right)=0
$$

and

$$
\sqrt{\varepsilon} u^{\prime}(-1)-k_{l}\left(u(-1)-s_{-}\right)=0
$$

studied in Ward [8]. 


\section{NUMBER OF SHOCKS}

As in our previous paper [6], our approach is based on the shooting method. That is, we start with the initial-value problem (IVP)

$$
\left\{\begin{array}{l}
\varepsilon u^{\prime \prime}+Q(u)=0 \\
u(-1)=0, \quad u^{\prime}(-1)=k,
\end{array}\right.
$$

where $k$ is a real number. When $Q(u)$ is sufficiently smooth, it is known that this problem has a unique solution which can be extended to all $x>-1$. For convenience, we introduce the notation

$$
k_{\max }:=\sqrt{\frac{2}{\varepsilon} \int_{0}^{s_{+}} Q(s) d s .}
$$

LEMMA 1. Let $u(x, k)$ denote the solution to the IVP (1.1) - (1.2). If $k>k_{\max }$, then $u(x, k)$ is increasing for $x>-1$ and $\lim _{x \rightarrow \infty} u(x, k)=\infty$. (b) If $k<-k_{\max }$, then $u(x, k)$ is decreasing for $x>-1$ and $\lim _{x \rightarrow \infty} u(x, k)=-\infty$. (c) If $|k|<k_{\max }$, then $u(x, k)$ is periodic and intersects the $x$-axis infinitely many times. (d) In the critical case, i.e. when $k= \pm k_{\max }, u(x, \varepsilon)= \pm f(\xi)$ where $\xi=\frac{x+1}{\sqrt{\varepsilon}}$ and $f$ satisfies

$$
\begin{gathered}
f^{\prime \prime}(\xi)+Q(f)=0, \quad-\infty<\xi<\infty, \\
f(-\infty)=s_{-}, \quad f(\infty)=s_{+}, \quad f(0)=0 ;
\end{gathered}
$$

furthermore,

$$
f(\xi) \sim s_{+}-A_{+} e^{-\sigma_{+} \xi}, \quad \text { as } \quad \xi \rightarrow \infty
$$

and

$$
f(\xi) \sim s_{-}+A_{-} e^{\sigma_{-} \xi}, \quad \text { as } \quad \xi \rightarrow-\infty
$$

where $\sigma_{ \pm}=\left(-Q^{\prime}\left(s_{ \pm}\right)\right)^{1 / 2}$, and $A_{+}, A_{-}$are explicitly given positive constants.

Proof. (a) Multiplying both sides of the differential equation in (2.1) by $u^{\prime}$, and integrating from -1 to $x$, we obtain

$$
\frac{\varepsilon}{2}\left(u^{\prime}\right)^{2}+\int_{0}^{u} Q(s) d s=C,
$$

where $C=\frac{\varepsilon}{2} k^{2}$. If $k>k_{\max }$, then it is easily seen from the graph of $Q(s)$ that $C>\int_{0}^{u} Q(s) d s$ for any $u>0$ and $\frac{\varepsilon}{2}\left(u^{\prime}\right)^{2}>0$; cf. (2.2). Since $u^{\prime}(-1, \varepsilon)=k>0$ and $u^{\prime}(x, \varepsilon)$ never vanishes, it follows that $u^{\prime}(x, \varepsilon)>0$ for $x>-1$. Therefore, $u(x, \varepsilon)$ is increasing for $x>-1$ and we can assume that

$$
\lim _{x \rightarrow \infty} u(x, \varepsilon)=l .
$$


We shall show that $l=\infty$. Since $k>k_{\max }$, there is a number $\beta>0$ such that

$$
k^{2}>\frac{2}{\varepsilon} \int_{0}^{s_{+}} Q(s) d s+\beta .
$$

Substituting this inequality into (2.7) gives

$$
u^{\prime}(x)>\sqrt{\beta}
$$

for $x>-1$, from which it follows that

$$
\lim _{x \rightarrow \infty} u(x, \varepsilon)=\infty .
$$

(b) The argument in this case is similar to that in case (a). Using (2.7), one can easily show that $u(x, \varepsilon)$ is decreasing for $x>-1$, and that it tends to $-\infty$ as $x$ approaches $\infty$.

(c) We consider only the case $0<k<k_{\max }$; the argument for $-k_{\max }<k<0$ is more or less the same. From the graph of $Q(u)$ in Figure 1, it is readily seen that the equation $\int_{0}^{u} Q(s) d s=C$ has two roots $u_{1}(k)$ and $u_{2}(k)$ such that $u_{1}(k) \in\left(0, s_{+}\right)$and $u_{2}(k) \in\left(s_{-}, 0\right)$. From the initial conditions in (2.1) and equation (2.7), it also follows that there are two points $x=d_{1}$ and $x=d_{2}$ with $d_{1}<d_{2}$ such that

$$
u^{\prime}\left(d_{1}, k\right)=0, \quad u\left(d_{1}, k\right)=u_{1}(k) \in\left(0, s_{+}\right),
$$

and

$$
u\left(d_{2}, k\right)=0, \quad u^{\prime}\left(d_{2}, k\right)=-k .
$$

Starting from $x=d_{2}$, one can also show that there exist two points $d_{3}$ and $d_{4}$ such that

$$
u^{\prime}\left(d_{3}, k\right)=0, \quad u\left(d_{3}, k\right)=u_{2}(k) \in\left(s_{-}, 0\right),
$$

and

$$
u\left(d_{4}, k\right)=0, \quad u^{\prime}\left(d_{4}, k\right)=k .
$$

At $x=d_{4}, u(x, k)$ has the same initial values as those at the starting point $x=-1$. Hence, $u(x, k)$ is periodic with period $d_{4}+1$.

In the case $k=0, u \equiv 0$ is the only solution to (2.1).

(d) When $|k|=k_{\max }$, we have $C=\frac{\varepsilon}{2} k^{2}=\int_{0}^{s_{+}} Q(s) d s$. First, consider the case $k=k_{\max }$, and make the change of variables

$$
\xi=\frac{x+1}{\sqrt{\varepsilon}}, \quad f(\xi)=u(x, k) .
$$

It is readily verified that $f$ satisfies (2.3). From (2.7), it also follows that

$$
\frac{1}{2}\left(\frac{d f}{d \xi}\right)^{2}+\int_{0}^{f} Q(s) d s=\int_{0}^{s_{+}} Q(s) d s .
$$

We claim

$$
0<f<s_{+}
$$


for $\xi>0$ and

$$
\lim _{\xi \rightarrow \infty} f(\xi)=s_{+} \cdot
$$

Since $f(0)=u(-1, k)=0$, it is easily seen that $f(\xi)<s_{+}$for $\xi$ in a right neighborhood of 0 . From (2.9), it can also be seen that there is such a neighborhood in which $f^{\prime}(\xi)>0$, i.e., $f(\xi)$ is increasing. We shall show that in fact, $f(\xi)$ is increasing in the whole interval $0<\xi<\infty$. Assume to the contrary that there exists a point $\eta$ such that $f(\eta)=s_{+}$and $0<f(\xi)<s_{+}$for $0<\xi<\eta$. Put

$$
V(f):=\int_{s_{+}}^{f} Q(s) d s .
$$

Clearly, $V\left(s_{+}\right)=V^{\prime}\left(s_{+}\right)=0$ and $V^{\prime \prime}\left(s_{+}\right)=Q^{\prime}\left(s_{+}\right)<0$. Since $V(f)$ is sufficiently smooth, we may write $V(f)=\left(s_{+}-f\right)^{2} H(f)$, where $H(f)$ is a negative function, so that

$$
\sigma_{+}=\sqrt{-Q^{\prime}\left(s_{+}\right)}=\sqrt{-2 H\left(s_{+}\right)} .
$$

(Note that as long as $\xi<\eta$, we have $f<s_{+}$and $V<0$.) From $(2.9)$, we obtain

$$
\frac{d f}{\left(s_{+}-f\right) \sqrt{-2 H(f)}}=d \xi .
$$

Integrating both sides from $\xi=0$ to $\xi=\eta$ gives

$$
\int_{0}^{s_{+}} \frac{d f}{\left(s_{+}-f\right) \sqrt{-2 H(f)}}=\int_{0}^{\eta} d \xi,
$$

which is not possible since the left-hand side is infinite while the right-hand is finite; this completes the proof of (2.10). Since $f^{\prime}(\xi)$ is positive in a right neighborhood of the origin and does not vanish elsewhere, it follows from $(2.9)$ that $f^{\prime}(\xi)>0$, i.e., $f(\xi)$ is increasing, in $0<\xi<\infty$ and

$$
\lim _{\xi \rightarrow \infty} f(\xi)=l \leq s_{+} .
$$

To show that $l=s_{+}$, we integrate (2.14) on both sides form 0 to $\infty$ to yield

$$
\int_{0}^{l} \frac{d f}{\left(s_{+}-f\right) \sqrt{-2 H(f)}}=\infty
$$

from which it follows that $l=s_{+}$, thus proving (2.11).

To derive the asymptotic behavior of $f(\xi)$, we return to (2.14) and obtain

$$
\int_{0}^{f} \frac{d s}{\left(s_{+}-s\right) \sqrt{-2 H(s)}}=\xi .
$$

The integral on the left-hand side can be written as

$$
\begin{aligned}
\int_{0}^{f} \frac{d s}{\left(s_{+}-s\right) \sqrt{-2 H\left(s_{+}\right)}} & +\int_{0}^{s_{+}} \frac{1}{\sqrt{2}\left(s_{+}-s\right)}\left(\frac{1}{\sqrt{-H(s)}}-\frac{1}{\sqrt{-H\left(s_{+}\right)}}\right) d s \\
& -\int_{f}^{s_{+}} \frac{1}{\sqrt{2}\left(s_{+}-s\right)}\left(\frac{1}{\sqrt{-H(s)}}-\frac{1}{\sqrt{-H\left(s_{+}\right)}}\right) d s .
\end{aligned}
$$


On account of (2.13), this gives

$$
\int_{0}^{f} \frac{d s}{\left(s_{+}-s\right) \sqrt{-2 H(s)}}=\frac{1}{\sigma_{+}}\left[\ln \left(s_{+}\right)-\ln \left(s_{+}-f\right)\right]+c_{+}-c_{2}(f),
$$

where

$$
c_{+}=\int_{0}^{s_{+}}\left(\frac{1}{\sqrt{-2 V(s)}}-\frac{1}{\left(s_{+}-s\right) \sigma_{+}}\right) d s
$$

and

$$
c_{2}(f)=\int_{f}^{s_{+}} \frac{1}{\sqrt{2}\left(s_{+}-s\right)}\left(\frac{1}{\sqrt{-H(s)}}-\frac{1}{\sqrt{-H\left(s_{+}\right)}}\right) d s .
$$

Note that the last two integrals are well defined. Coupling (2.16) and (2.17), we have

$$
f=s_{+}-s_{+} e^{\sigma_{+} c_{+}} e^{-\sigma_{+} \xi} e^{-c_{2}(f) \sigma_{+}} .
$$

Since $c_{2}(f) \rightarrow 0$ as $f \rightarrow s_{+}$, the result in (2.5) follows with $A_{+}=s_{+} e^{\sigma_{+} c_{+}}$. A similar argument can be used to demonstrate (2.6) with $A_{-}=-s_{-} e^{\sigma_{-} c_{-}}$where

$$
c_{-}=\int_{0}^{s_{-}}\left(\frac{-1}{\sqrt{-2 V(s)}}-\frac{1}{\left(s_{-}-s\right) \sigma_{-}}\right) d s
$$

This completes the proof of the lemma.

REMARK 1. The proof of case (d) above actually gives the existence of a monotonically increasing function $f(\xi)$, which satisfies the differential equation in (2.3) and the conditions in (2.4). Furthermore, it has the asymptotic behavior given in (2.5) and (2.6). This result is stated explicitly in Ward [8, p.99], but without a proof.

REMARK 2. Using (2.13) and (2.14), one can also deduce from (2.5) and (2.6)

$$
\begin{aligned}
& f^{\prime}(\xi) \sim \sigma_{+} A_{+} e^{-\sigma_{+} \xi}, \quad \text { as } \quad \xi \rightarrow \infty, \\
& f^{\prime}(\xi) \sim \sigma_{-} A_{-} e^{\sigma_{-} \xi}, \quad \text { as } \quad \xi \rightarrow-\infty .
\end{aligned}
$$

From Lemma 1, it is clear that in order to have $u(1, k)=0$, we must choose $k$ in the interval $\left(-k_{\max }, 0\right)$ or $\left(0, k_{\max }\right)$, in which case the solution to the initial-value problem $(2.1)$ is periodic and intersects the $x$-axis infinitely many times.

We consider only the case $k \in\left(0, k_{\max }\right)$, since the case $k \in\left(-k_{\max }, 0\right)$ is entirely similar. Let $x_{1}$ be the first point to the right of -1 such that $u\left(x_{1}, k\right)=0$ and $u(x, k)>0$ for $-1<x<x_{1}$, and $x_{2}$ be the first point to the right of $x_{1}$ such that $u\left(x_{2}, k\right)=0$ and $u(x, k)<0$ for $x_{1}<x<x_{2}$. We denote by $T_{1}(k)$ and $T_{2}(k)$, respectively, the lengths of the interval $\left[-1, x_{1}\right]$ and $\left[x_{1}, x_{2}\right]$. From $(2.7)$ and the graph of $Q(u)$ in Figure 1, we can also find two points $u_{1}(k) \in\left(0, s_{+}\right)$and $u_{2}(k) \in\left(s_{-}, 0\right)$ such that

$$
-\int_{u_{2}(k)}^{0} Q(s) d s=\int_{0}^{u_{1}(k)} Q(s) d s=\frac{\varepsilon}{2} k^{2} .
$$


It is readily seen that when $k \rightarrow 0, u_{1}(k)$ and $u_{2}(k)$ tend to zero and when $k \rightarrow k_{\max }, u_{1}(k)$ tends to $s_{+}$and $u_{2}(k)$ tends to $s_{-}$. Differentiating both sides of $(2.22)$ with respect to $k$ shows that $u_{1}^{\prime}(k)>0$ and $u_{2}^{\prime}(k)<0$. From $(2.7)$, we obtain

$$
\sqrt{\varepsilon} \frac{d u}{\sqrt{2 \int_{u}^{u_{1}(k)} Q(s) d s}}=d x
$$

from which it follows that

$$
T_{1}(k)=\sqrt{2 \varepsilon} \int_{0}^{u_{1}(k)} \frac{d u}{\sqrt{\int_{u}^{u_{1}(k)} Q(s) d s}}
$$

and

$$
T_{2}(k)=\sqrt{2 \varepsilon} \int_{u_{2}(k)}^{0} \frac{d u}{\sqrt{\int_{u}^{u_{2}(k)} Q(s) d s}} .
$$

In the case $k \in\left(-k_{\max }, 0\right)$, the solution $u(x, k)$ to the initial-value problem (2.1) is again oscillatory about the $x$-axis, except that it now starts at $x=-1$ with a negative slope. Hence, the graph of $u(x, k)$ is below the $x$-axis in the interval $\left(-1, x_{1}\right)$, where $x_{1}$ is the first point to the right of -1 at which $u(x, k)$ meets the line $u=0$ again, i.e., $u(x, k)<0$ in $\left(-1, x_{1}\right)$. Let $x_{2}$ denote the first point to the right of $x_{1}$ such that $u\left(x_{2}, k\right)=0$ and $u(x, k)>0$ for $x_{1}<x<x_{2}$. Unlike before, we now reverse the order, and denote by $T_{2}(k)$ and $T_{1}(k)$ the length of the intervals $\left[-1, x_{1}\right]$ and $\left[x_{1}, x_{2}\right]$, respectively. Hence, the values of $T_{1}(k)$ and $T_{2}(k)$ are actually dependent on the value of $|k|$.

LEMMA 2. We have

$$
\lim _{k \rightarrow 0^{+}} T_{1}(k)=\lim _{k \rightarrow 0^{+}} T_{2}(k)=\frac{\pi \sqrt{\varepsilon}}{\sqrt{Q^{\prime}(0)}} .
$$

Proof. Let

$$
V_{2}(u)=\int_{0}^{u} Q(s) d s .
$$

Since $V_{2}(u)$ is sufficiently smooth and $V_{2}(0)=V_{2}^{\prime}(0)=0$, we may write $V_{2}(u)=u^{2} G(u)$, where $G$ is a sufficiently smooth function with $G(0)=\frac{1}{2} Q^{\prime}(0)$. From (2.24), it follows that

$$
\begin{aligned}
T_{1}(k) & =\sqrt{2 \varepsilon} \int_{0}^{u_{1}(k)} \frac{d u}{\sqrt{V_{2}\left(u_{1}(k)\right)-V_{2}(u)}} \\
& =\sqrt{2 \varepsilon} \int_{0}^{u_{1}(k)} \frac{d u}{\sqrt{u_{1}^{2}(k) G\left(u_{1}(k)\right)-u^{2} G(u)}} .
\end{aligned}
$$

It is easily seen that

$$
\sqrt{2 \varepsilon} \int_{0}^{u_{1}(k)} \frac{d u}{\sqrt{G(0)\left(u_{1}^{2}(k)-u^{2}\right)}}=\frac{\pi}{2} \frac{\sqrt{2 \varepsilon}}{\sqrt{G(0)}}=\frac{\pi \sqrt{\varepsilon}}{\sqrt{Q^{\prime}(0)}} .
$$


Since the integral

$$
\sqrt{2 \varepsilon} \int_{0}^{u_{1}(k)}\left(\frac{1}{\sqrt{V_{2}\left(u_{1}(k)\right)-V_{2}(u)}}-\frac{1}{\sqrt{G(0)\left(u_{1}^{2}(k)-u^{2}\right)}}\right) d u .
$$

exists and the length of the integration interval tends to zero as $k \rightarrow 0$, we obtain

$$
T_{1}(k)-\frac{\pi \sqrt{\varepsilon}}{\sqrt{Q^{\prime}(0)}} \rightarrow 0 \quad \text { as } \quad k \rightarrow 0
$$

In a similar manner, one can prove

$$
\lim _{k \rightarrow 0^{+}} T_{2}(k)=\frac{\pi \sqrt{\varepsilon}}{\sqrt{Q^{\prime}(0)}} .
$$

On the other hand, we have $u_{1}(k) \rightarrow s_{+}$and $u_{2}(k) \rightarrow s_{-}$as $k \rightarrow k_{\max }$; see the second paragraph following Remark 2. Hence

$$
\lim _{k \rightarrow k_{\max }} T_{1}(k)=\lim _{k \rightarrow k_{\max }} T_{2}(k)=\infty .
$$

Put

$$
t_{1}:=\inf _{0<k<k_{\max }} T_{1}(k)
$$

and

$$
t_{2}:=\inf _{0<k<k_{\max }} T_{2}(k)
$$

It is easily seen that

$$
0<t_{1}, t_{2} \leq \frac{\pi \sqrt{\varepsilon}}{\sqrt{Q^{\prime}(0)}}
$$

Recall that our goal is to use the shooting method to determine whether the original boundary-value problem has solutions with shocks. If it has, then we would like to give an estimate to the number of shocks that a solution can have. This is equivalent to choosing appropriate values of $k$ so that

$$
u(1, k)=0 .
$$

Since $u(x, k)$ is periodic and oscillatory, equation (2.31) can be expressed in the form

$$
N T_{1}(k)+M T_{2}(k)=2,
$$

where $N$ and $M$ are nonnegative integers and represent, respectively, the number of intervals with lengths $T_{1}(k)$ and $T_{2}(k)$. Now we look at the arches bounded by the graph of $u(x, k)$ and the line $u=0$. Since every arch above the line $u=0$ is followed by an arch below $u=0$ and vice versa, the integers $N$ and $M$ are related in such a way that for every given $N$, there are only three possible values of $M$, namely, $M=N-1, N$, or $N+1$. It is easy to see 
that the number of zeros, which corresponds to the number of shocks, of a solution $u(x, k)$ in the interval $(-1,1)$ is $N+M-1$. Therefore, the number of shock layers is $N+M-1$.

From here on, our task is to choose appropriate values of $k$, and determine possible integers $N$ and $M$, so that (2.32) holds.

For $\varepsilon>0$, we define

$$
N_{1}(\varepsilon):=\left[\frac{2}{t_{1}+t_{2}}\right]
$$

and

$$
N_{2}(\varepsilon):=\left[\frac{2}{T_{1}\left(0_{+}\right)+T_{2}\left(0_{+}\right)}\right],
$$

where $t_{1}, t_{2}$ are given above and $[x]$ denotes the largest integer less than or equal to $x$. The following result provides estimates for the number of shocks that the solutions to BVP (1.1)-(1.2) can have.

LEMMA 3. If $N \geq N_{1}(\varepsilon)+1$ and $M \geq N_{1}(\varepsilon)+1$, then there exists no slope $k$ such that (2.32) holds, i.e., the BVP (1.1) - (1.2) has no solution with more than or equal to $2 N_{1}(\varepsilon)+1$ shocks. If $N \leq N_{2}(\varepsilon)$ and $M \leq N_{2}(\varepsilon)$, then there exists at least one value of $k$ so that (2.32) holds, i.e., BVP (1.1) - (1.2) has at least one solution with $N+M-1$ shocks.

Proof. The function

$$
N T_{1}(k)+M T_{2}(k)
$$

is continuous in $0<k<k_{\max }$. For any $k>0$, we have by (2.33)

$$
\begin{aligned}
N T_{1}(k)+M T_{2}(k) & \geq N t_{1}+M t_{2} \\
& \geq\left(N_{1}(\varepsilon)+1\right) t_{1}+\left(N_{1}(\varepsilon)+1\right) t_{2} \\
& =\left(N_{1}(\varepsilon)+1\right)\left(t_{1}+t_{2}\right)>2 .
\end{aligned}
$$

Thus, (2.32) fails to hold and the boundary-value problem has no solution.

If $N \leq N_{2}(\varepsilon)$ and $M \leq N_{2}(\varepsilon)$, then by (2.34) and (2.27) the function $N T_{1}(k)+M T_{2}(k)$ satisfies

$$
\lim _{k \rightarrow 0_{+}} N T_{1}(k)+M T_{2}(k) \leq N_{2}(\varepsilon)\left(T_{1}\left(0_{+}\right)+T_{2}\left(0_{+}\right)\right) \leq 2
$$

and

$$
\lim _{k \rightarrow k_{\max }} N T_{1}(k)+M T_{2}(k)=\infty .
$$

In view of the continuity of the function $N T_{1}(k)+M T_{2}(k)$, there exists at least one point $k_{0} \in\left(0, k_{\max }\right)$ such that

$$
N T_{1}\left(k_{0}\right)+M T_{2}\left(k_{0}\right)=2,
$$

which means that there exists at least one solution to BVP (1.1)-(1.2). Also, it follows that this solution has $N+M-1$ zeros, and hence $N+M-1$ internal shock layers, in the interval $(-1,1)$.

When $Q(u)$ satisfies the additional condition given below, we can in fact establish the monotonicity of $T_{1}(k)$ and $T_{2}(k)$ with respect to $k$, in which case more accurate estimates for the numbers of shocks and solutions can be obtained. 
LEMMA 4. If, in addition, $Q(s) / s$ is decreasing in $s$ for $s \in\left(0, s_{+}\right)$and increasing for $s \in\left(s_{-}, 0\right)$, then $T_{1}(k)$ and $T_{2}(k)$ are increasing in $k$ for $k \in\left(0, k_{\max }\right)$.

Proof. From (2.24), we have

$$
T_{1}(k)=\sqrt{2 \varepsilon} \int_{0}^{u_{1}(k)} \frac{d u}{\sqrt{\int_{u}^{u_{1}(k)} Q(s) d s}} .
$$

Changing variables $u=u_{1}(k) t$ yields

$$
T_{1}(k)=\sqrt{2 \varepsilon} \int_{0}^{1} \frac{d t}{\sqrt{f_{1}(k)}},
$$

where

$$
f_{1}(k)=\frac{1}{u_{1}^{2}(k)} \int_{u_{1}(k) t}^{u_{1}(k)} Q(s) d s .
$$

Straightforward differentiation gives

$$
\frac{d f_{1}}{d u_{1}}=\frac{1}{u_{1}^{3}}\left(Q\left(u_{1}\right) u_{1}-2 \int_{0}^{u_{1}} Q(s) d s-\left[Q\left(t u_{1}\right) t u_{1}-2 \int_{0}^{t u_{1}} Q(s) d s\right]\right) .
$$

Since $Q(x) / x$ is decreasing, we have $Q^{\prime}(x) x-Q(x)<0$ for almost all $x \in\left(0, s_{+}\right)$, from which it follows that $Q(x) x-2 \int_{0}^{x} Q(s) d s$ is decreasing. Hence

$$
\frac{d f_{1}}{d u_{1}}<0 \quad \text { and } \quad \frac{d f_{1}}{d k}=\frac{d f_{1}}{d u_{1}} \frac{d u_{1}}{d k}<0
$$

for almost all $k \in\left(0, k_{\max }\right)$; see a statement following $(2.22)$. Note that $d T_{1} / d f_{1}$ is negative. Thus, by the chain rule,

$$
\frac{d T_{1}(k)}{d k}>0
$$

i.e., $T_{1}(k)$ is increasing in $k$. The proof of the monotonicity of $T_{2}(k)$ is similar; this completes the proof of the lemma.

From Lemma 4, it follows that

$$
t_{1}=t_{2}=T_{1}\left(0_{+}\right)=T_{2}\left(0_{+}\right)=\frac{\pi \sqrt{\varepsilon}}{\sqrt{Q^{\prime}(0)}} .
$$

REMARK 3. Note that the function $s Q^{\prime}(s)-Q(s)$ vanishes at $s=0$, is negative in $\left(0, s_{+}\right)$and positive in $\left(s_{-}, 0\right)$. Hence, if $Q(s)$ satisfies $Q^{\prime \prime}(s)<0$ for $s \in\left(0, s_{+}\right)$and $Q^{\prime \prime}(s)>0$ for $s \in\left(0, s_{-}\right)$, then the condition in Lemma 4 holds and $T_{1}(k), T_{2}(k)$ are increasing in $k$ for $0<k<k_{\max }$.

Let

$$
N(\varepsilon)=\left[\frac{2}{t_{1}}\right]=\left[\frac{2 \sqrt{Q^{\prime}(0)}}{\pi \sqrt{\varepsilon}}\right] .
$$

We have the following result concerning the numbers of shocks and solutions to the BVP $(1.1)-(1.2)$ 
THEOREM 1. Let $n_{1}(\varepsilon)$ denote the numbers of shocks that a solution to (1.1)-(1.2) can have, and let $n_{2}(\varepsilon)$ denote the number of solutions to (1.1)-(1.2). If $Q(s) / s$ is decreasing for $s \in\left(0, s_{+}\right)$and increasing for $s \in\left(s_{-}, 0\right)$, then $n_{1}(\varepsilon)$ satisfies

$$
0 \leq n_{1}(\varepsilon) \leq N(\varepsilon)-1
$$

and $n_{2}(\varepsilon)$ satisfies

$$
n_{2}(\varepsilon)=2 N(\varepsilon)
$$

where $N(\varepsilon)$ is given in (2.36).

Proof. If $n_{1}(\varepsilon) \leq N(\varepsilon)-1$, we choose

$$
M=\left[\frac{n_{1}(\varepsilon)+1}{2}\right]
$$

and

$$
N=n_{1}(\varepsilon)-\left[\frac{n_{1}(\varepsilon)+1}{2}\right]+1
$$

Then $N \geq M, N+M=n_{1}(\varepsilon)+1 \leq N(\varepsilon)$, and

$$
N-M \leq 1
$$

Consider the function

$$
N T_{1}(k)+M T_{2}(k)
$$

By (2.35) and (2.36), we have

$$
\begin{aligned}
& \lim _{k \rightarrow 0_{+}} N T_{1}(k)+M T_{2}(k)=(N+M) t_{1} \\
= & \left(n_{1}(\varepsilon)+1\right) t_{1} \leq N(\varepsilon) t_{1} \leq 2
\end{aligned}
$$

and

$$
\lim _{k \rightarrow k_{\max }} N T_{1}(k)+M T_{2}(k)=\infty .
$$

Since by Lemma 4 this function is increasing in $k$, there exists a unique point $k_{0}$ such that $N T_{1}\left(k_{0}\right)+M T_{2}\left(k_{0}\right)=2$.

If $n_{1}(\varepsilon) \geq N(\varepsilon)$, then $N+M \geq n_{1}(\varepsilon)+1 \geq N(\varepsilon)+1$ and by (2.28) and (2.36)

$$
N T_{1}(k)+M T_{2}(k) \geq(N+M) t_{1} \geq(N(\varepsilon)+1) t_{1}>2
$$

for any $k$. Thus, we cannot expect $(2.32)$ to hold, i.e., there exists no solution to problem $(1.1)-(1.2)$ if $n_{1}(\varepsilon) \geq N(\varepsilon)$.

On one hand, for any $n_{1}(\varepsilon)$ in $0 \leq n_{1}(\varepsilon) \leq N(\varepsilon)-1$, we can choose $M$ and $N$ as given in (2.37) and (2.38) so that there exists exactly one value $k_{0}$ satisfying $u\left(1, k_{0}\right)=0$. On the other hand, by interchanging the two values of $M$ and $N$ in (2.37) and (2.38) and 
considering $k \in\left(-k_{\max }, 0\right)$, we conclude by the symmetry that $u\left(1,-k_{0}\right)=0$. Therefore, there are exactly two solutions with $n_{1}(\varepsilon)$ shock layers.

Adding all solutions together, we get the number of solutions to (1.1) - (1.2) given by

$$
n_{2}(\varepsilon)=2 N(\varepsilon)
$$

This completes the proof of the theorem.

When $Q(u)=2 u-2 u^{3}, Q(u) / u=2-2 u^{2}$ is decreasing for $u>0$ and increasing for $u<0$. Therefore, Lemma 4 applies and both $T_{1}(k)$ and $T_{2}(k)$ are increasing in $k$ for $k>0$. For $k<0$, the situation is just the opposite and both $T_{1}(k)$ and $T_{2}(k)$ are decreasing in $k$ for $k<0$. In fact, $T_{1}(k)$ and $T_{2}(k)$ depend on the absolute value of $k$ (i.e., $|k|$ ), and we have

$$
T_{1}(k)=T_{1}(-k), \quad T_{2}(k)=T_{2}(-k) .
$$

From (2.35) and (2.36), it follows that

$$
t_{1}=\inf _{k>0} T_{1}(k)=T_{1}\left(0_{+}\right)=\frac{\sqrt{\varepsilon} \pi}{\sqrt{2}}
$$

and

$$
N(\varepsilon)=\left[\frac{2}{t_{1}}\right]=\left[\frac{2 \sqrt{2}}{\sqrt{\varepsilon} \pi}\right] .
$$

Applying Theorem 1, we obtain the following result.

COROLLARY 1. If $Q(u)=2 u-2 u^{3}$, then the number $n_{1}(\varepsilon)$ of shocks, that a solution to problem $(1.1)-(1.2)$ can have, satisfies

$$
0 \leq n_{1}(\varepsilon) \leq N(\varepsilon)-1=\left[\frac{2 \sqrt{2}}{\sqrt{\varepsilon} \pi}\right]-1 .
$$

Moreover, the number $n_{2}(\varepsilon)$ of solutions to (1.1) - (1.2) satisfies

$$
n_{2}(\varepsilon)=2 N(\varepsilon)=2\left[\frac{2 \sqrt{2}}{\sqrt{\varepsilon} \pi}\right] \sim \frac{4 \sqrt{2}}{\sqrt{\varepsilon} \pi} .
$$

If $Q(u)=\sin (\pi u)$, then $s(Q(s) / s)^{\prime} \leq 0$ for $s \in[-1,1], Q^{\prime}(0)=\pi$ and $N(\varepsilon)=\left[\frac{2}{\sqrt{\pi \varepsilon}}\right]$. A similar result is obtained.

COROLLARY 2. If $Q(u)=\sin (\pi u)$, then the number $n_{1}(\varepsilon)$ of shocks, that a solution to problem $(1.1)-(1.2)$ can have, satisfies

$$
0 \leq n_{1}(\varepsilon) \leq N(\varepsilon)-1=\left[\frac{2}{\sqrt{\pi \varepsilon}}\right]-1 .
$$

Moreover, the number $n_{2}(\varepsilon)$ of solutions to (1.1) - (1.2) satisfies

$$
n_{2}(\varepsilon)=2 N(\varepsilon)=2\left[\frac{2}{\sqrt{\pi \varepsilon}}\right] \sim \frac{4}{\sqrt{\varepsilon \pi}} .
$$




\section{APPROXIMATE SOLUTIONS WITH AT MOST ONE SHOCK}

First, we confine ourselves to solutions with no shock layers, i.e., solutions which is either entirely positive, or entirely negative, in the interval $(-1,1)$.

THEOREM 2. The BVP problem (1.1) - (1.2) has one and only one solution $u_{0,1}(x)$ which is positive in the interval $(-1,1)$. The asymptotic formula for this solution is given by

$$
u_{0,1}(x)=f\left(\frac{x+1}{\sqrt{\varepsilon}}\right)+f\left(\frac{1-x}{\sqrt{\varepsilon}}\right)-s_{+}+O\left(e^{-\sigma_{+} / \sqrt{\varepsilon}}\right)
$$

where $f$ is the function given in Lemma 1(d). Likewise, the problem (1.1) - (1.2) also has one and only one solution $u_{0,2}(x)$ which is negative in the interval $(-1,1)$. The asymptotic formula for this solution is given by

$$
u_{0,2}(x)=f\left(-\frac{x+1}{\sqrt{\varepsilon}}\right)+f\left(-\frac{1-x}{\sqrt{\varepsilon}}\right)-s_{-}+O\left(e^{-\sigma_{-} / \sqrt{\varepsilon}}\right) .
$$

Proof. We shall give only the proof of (3.1), as the proof of (3.2) is very similar. It is easily seen that in order to have a positive solution to the BVP (1.1)-(1.2), we must choose a solution to the IVP (2.1) with a positive slope $k$. First, we recall that if $Q(x) / x$ is decreasing in $x$ for $x \in\left(0, s_{+}\right)$then by Lemma $4, T_{1}(k)$ is increasing in $k$ with the endpoint values

$$
T_{1}\left(0_{+}\right)=O(\varepsilon)
$$

and

$$
\lim _{k \rightarrow k_{\max }} T_{1}(k)=\infty ;
$$

cf. Lemma 2 and (2.27). By the continuity of $T_{1}(k)$, we conclude that there exists one and only one value $k_{0}$ such that $T_{1}\left(k_{0}\right)=2$, i.e., equation (2.32) holds with $k=k_{0}, N=1$ and $M=0$. This, in turn, implies the existence of a unique positive solution $u\left(x, k_{0}\right)$ to the IVP (2.1) which also satisfies the boundary conditions in (1.2). We denote this solution by $u_{0,1}(x)$. Returning to the original equation, it is easily verified that $u_{0,1}(-x)$ is also a solution to (1.1)-(1.2). Using the uniqueness of solution, we have $u_{0,1}(x)=u_{0,1}(-x)$, which means the solution $u_{0,1}(x)$ is symmetric and $u_{0,1}^{\prime}(0)=0$. Furthermore, it is readily seen that the solution $u_{0,1}(x)$ is increasing in $[-1,0)$ and decreasing in $(0,1]$.

If $Q(x) / x$ is not decreasing in $x$, we can still establish the uniqueness result. To this end, we note that since $Q^{\prime}\left(s_{+}\right)<0$, and $Q\left(s_{+}\right)=0$, we can choose a constant $\delta>0$, which is independent of $\varepsilon$ such that for $x$ in the internal $\left(s_{+}-\delta, s_{+}\right)$

$$
Q(x)+x Q^{\prime}(x)<-m_{1},
$$

where $m_{1}$ is a positive number. Let $k_{\delta}$ satisfy

$$
\int_{0}^{s_{+}-\delta} Q(s) d s=\frac{\varepsilon}{2} k_{\delta}^{2} .
$$


For $k \in\left(0, k_{\delta}\right)$, we have $u_{1}(k) \in\left(0, s_{+}-\delta\right)$ since $u_{1}(k)$ is increasing in $\left(0, s_{+}\right)$, and

$$
T_{1}(k)=\sqrt{2 \varepsilon} \int_{0}^{u_{1}(k)} \frac{d u}{\sqrt{\int_{u}^{u_{1}(k)} Q(s) d s}}=O(\sqrt{\varepsilon})
$$

For convenience, we still denote by $k_{0}$ the minimum value of $k$ satisfying $T_{1}(k)=2$, namely

$$
k_{0}=\inf \left\{k: T_{1}(k)=2\right\} .
$$

From (2.27) and (3.4), it is clear that

$$
k_{0}>k_{\delta} .
$$

Since $u_{1}^{\prime}(k)>0$, it follows from $(2.22)$ that

$$
u_{1}\left(k_{0}\right)>u_{1}\left(k_{\delta}\right)=s_{+}-\delta
$$

see the statement between (2.22) and (2.23). Furthermore, since

$$
T_{1}\left(k_{0}\right)=2,
$$

we have from $(2.24)$

$$
\int_{0}^{u_{1}\left(k_{0}\right)} \frac{d u}{\sqrt{2} \sqrt{\left(s_{+}-u_{1}\right)^{2} H\left(u_{1}\right)-\left(s_{+}-u\right)^{2} H(u)}}=\sqrt{\frac{1}{\varepsilon}},
$$

where $u_{1}\left(k_{0}\right)$ is the maximum value that $u_{0,1}(x)$ attains at $x=0$. Let $t=s_{+}-u$ and $a=s_{+}-u_{1}\left(k_{0}\right)$. Then

$$
\int_{a}^{s_{+}} \frac{d t}{\sqrt{2} \sqrt{t^{2} g(t)-a^{2} g(a)}}=\sqrt{\frac{1}{\varepsilon}}
$$

where $g(t)=-H\left(s_{+}-t\right)>0$ for any $t \in\left[0, s_{+}\right]$and also satisfies

$$
t^{2} g(t)-a^{2} g(a)>0
$$

for $t \in\left(a, s_{+}\right)$and

$$
g(0)=-H\left(s_{+}\right)=-\frac{Q^{\prime}\left(s_{+}\right)}{2}, \quad g^{\prime}(0)=\frac{Q^{\prime \prime}\left(s_{+}\right)}{6} .
$$

Consider the function

$$
\frac{t^{2} g(t)-a^{2} g(a)}{t^{2}-a^{2}},
$$

which is positive and continuous in the interval $\left(a, s_{+}\right]$. When $t \rightarrow a$, using L'Hospital's rule, we have

$$
\begin{aligned}
\lim _{t \rightarrow a} \frac{t^{2} g(t)-a^{2} g(a)}{t^{2}-a^{2}} & =g(a)+\frac{1}{2} a g^{\prime}(a) \\
& =\frac{Q\left(s_{+}-a\right)}{2 a}>0
\end{aligned}
$$


for any $a=s_{+}-u_{1}\left(k_{0}\right)<s_{+}$. So there is a positive constant $m$ such that

$$
\frac{t^{2} g(t)-a^{2} g(a)}{t^{2}-a^{2}}>m
$$

for $t \in\left(a, s_{+}\right]$. By (3.6), we have from (3.5)

$$
\int_{a}^{s_{+}} \frac{d t}{\sqrt{2 m} \sqrt{t^{2}-a^{2}}}>\sqrt{\frac{1}{\varepsilon}}
$$

which yields

$$
\cosh ^{-1} \frac{s_{+}}{a}>\sqrt{\frac{2 m}{\varepsilon}}
$$

and hence

$$
a \leq 2 s_{+} e^{-\sqrt{2 m / \varepsilon}} .
$$

We now show that $T(k)$ is strictly increasing for $k \geq k_{0}$. This, in turn, gives the uniqueness of the positive solution to BVP (1.1)-(1.2).

To differentiate $T_{1}(k)$ with respect to $u_{1}(k)$, we first make the change of variable $u=$ $t u_{1}(k)$, and then use the product rule. After dropping the term with the positive sign, we obtain

$$
\frac{d T_{1}(k)}{d u_{1}(k)} \geq-\sqrt{\frac{\varepsilon}{2}} \int_{0}^{1}\left(\int_{u_{1}(k) t}^{u_{1}(k)} Q(s) d s\right)^{-3 / 2}\left[u_{1}(k) Q\left(u_{1}(k)\right)-t u_{1}(k) Q\left(t u_{1}(k)\right)\right] d t .
$$

On the right-hand side, we change the integration variable back to $u$, and write

$$
\frac{d T_{1}(k)}{d u_{1}(k)} \geq-\sqrt{\frac{\varepsilon}{2}} \frac{1}{u_{1}(k)}\left(I_{1}+I_{2}\right)
$$

where $I_{1}$ and $I_{2}$ are integrals of $\left(\int_{u}^{u_{1}(k)} Q(s) d s\right)^{-3 / 2}\left[u_{1}(k) Q\left(u_{1}(k)\right)-u Q(u)\right]$ on the intervals $\left(0, s_{+}-\delta\right)$ and $\left(s_{+}-\delta, u_{1}(k)\right)$, respectively. When $k \geq k_{0}$, the integrand of $I_{1}$ is bounded by some constant $M_{1}$. Thus,

$$
I_{1} \leq M_{1}\left(s_{+}-\delta\right) .
$$

On the interval $\left(s_{+}-\delta, u_{1}(k)\right)$, we have by the mean-value theorem and (3.3)

$$
\begin{aligned}
u_{1}(k) Q\left(u_{1}(k)\right)-u Q(u) & =\left(Q(\bar{u})+\bar{u} Q^{\prime}(\bar{u})\right)\left(u_{1}(k)-u\right) \\
& \leq-m_{1}\left(u_{1}(k)-u\right),
\end{aligned}
$$

where $u \leq \bar{u} \leq u_{1}(k)$. When $k \geq k_{0}, u_{1}(k) \geq u_{1}\left(k_{0}\right) \geq s_{+}-a$. Substituting (3.8) into $I_{2}$, and letting $t=s_{+}-u$, and $\bar{a}=s_{+}-u_{1}(k)$, where $\bar{a}<a$ when $k \geq k_{0}$, we get

$$
I_{2} \leq-m_{1} \int_{\bar{a}}^{\delta} \frac{t-\bar{a}}{\left(t^{2} g(t)-\bar{a}^{2} g(\bar{a})\right)^{3 / 2}} d t \leq-m_{1} m_{2} \cosh ^{-1} \frac{\delta}{\bar{a}}
$$

Here, we have made use of the fact that

$$
\frac{t-\bar{a}}{\left(t^{2} g(t)-\bar{a}^{2} g(\bar{a})^{3 / 2}\right.} \geq m_{2} \frac{1}{\sqrt{t^{2}-\bar{a}^{2}}}
$$


for $t \in[\bar{a}, \delta]$. Hence,

$$
I_{1}+I_{2}<0 \quad \text { when } \quad \bar{a} \leq a \leq 2 s_{+} e^{-\sqrt{2 m / \varepsilon}} .
$$

Since $u_{1}^{\prime}(k)>0$, we have

$$
\frac{d T_{1}(k)}{d k}=\frac{d T_{1}(k)}{d u_{1}(k)} \frac{d u_{1}(k)}{d k}>0
$$

for $k \geq k_{0}$. This completes the proof of the uniqueness.

Next, we will prove (3.1). In (3.7), we already have a rough estimate for $a$, which states that $a \rightarrow 0$ as $\varepsilon \rightarrow 0$. Using this piece of information, we shall now give a more refined estimate. The left-hand side of (3.5) can be written as

$$
\int_{a}^{s_{+}} \frac{d t}{\sqrt{2} \sqrt{t^{2} g(t)-a^{2} g(a)}}=\int_{a}^{s_{+}} \frac{d t}{\sqrt{2} \sqrt{\left(t^{2}-a^{2}\right) F(t, a)}}
$$

where

$$
F(t, a)=\frac{t^{2} g(t)-a^{2} g(a)}{t^{2}-a^{2}} .
$$

From (3.6), it is clear that for any $a \in\left(0, s_{+}\right), F(t, a)$ is positive and continuous for $t>a$. Moreover, we have

$$
\lim _{t \rightarrow a} F(t, a)=g(a)+\frac{a}{2} g^{\prime}(a)
$$

and

$$
\lim _{t \rightarrow a} \frac{\partial F(t, a)}{\partial t}=\frac{3}{4} g^{\prime}(a)+\frac{1}{4} a g^{\prime \prime}(a) .
$$

From (3.9), we obtain

$$
\begin{aligned}
& \int_{a}^{s_{+}} \frac{d t}{\sqrt{2} \sqrt{t^{2} g(t)-a^{2} g(a)}} \\
& =\frac{1}{\sqrt{2 g(a)+a g^{\prime}(a)}} \int_{a}^{s_{+}} \frac{d t}{\sqrt{t^{2}-a^{2}}}+G_{1}(a),
\end{aligned}
$$

where

$$
G_{1}(a)=\int_{a}^{s_{+}} \frac{1}{\sqrt{\left(t^{2}-a^{2}\right)}}\left(\frac{1}{\sqrt{2 F(t, a)}}-\frac{1}{\sqrt{2 g(a)+a g^{\prime}(a)}}\right) d t .
$$

The first term on the right-hand side of (3.10) can be evaluated explicitly; to the second term, we apply the mean-value theorem. This gives

$$
\begin{aligned}
\int_{a}^{s_{+}} \frac{d t}{\sqrt{2} \sqrt{t^{2} g(t)-a^{2} g(a)}} & =\frac{1}{\sqrt{2 g(a)+a g^{\prime}(a)}} \cosh ^{-1} \frac{s_{+}}{a} \\
& +\int_{0}^{s_{+}} \frac{1}{\sqrt{t^{2}}}\left(\frac{1}{\sqrt{2 F(t, 0)}}-\frac{1}{\sqrt{2 g(0)}}\right) d t+O(a) .
\end{aligned}
$$

Since $g(0)=-Q^{\prime}\left(s_{+}\right) / 2=\sigma_{+}^{2}$ and $g^{\prime}(0)=Q^{\prime \prime}\left(s_{+}\right) / 6$, it can be shown that

$$
\frac{1}{\sqrt{2 g(a)+a g^{\prime}(a)}} \cosh ^{-1} \frac{s_{+}}{a}=\frac{1}{\sqrt{2 g(0)}}\left(\ln \frac{s_{+}}{a}+\ln 2\right)+\frac{Q^{\prime \prime}\left(s_{+}\right)}{4 \sigma_{+}^{3}} a \ln a+O(a)
$$


and the last integral is equal to the integral in (2.18). Thus

$$
\int_{a}^{s_{+}} \frac{d t}{\sqrt{2} \sqrt{t^{2} g(t)-a^{2} g(a)}}=\frac{1}{\sigma_{+}}\left(\ln \frac{s_{+}}{a}+\ln 2\right)+c_{+}+\frac{Q^{\prime \prime}\left(s_{+}\right)}{4 \sigma_{+}^{3}} a \ln a+O(a)
$$

Inserting the above formula into (3.5), we obtain

$$
\begin{aligned}
a & =2 s_{+} e^{\sigma_{+} c_{+}} e^{-\sigma_{+} / \sqrt{\varepsilon}}\left(1+\frac{Q^{\prime \prime}\left(s_{+}\right) A_{+}}{2 \sigma_{+} \sqrt{\varepsilon}} e^{-\sigma_{+} / \sqrt{\varepsilon}}+O\left(e^{-\sigma_{+} / \sqrt{\varepsilon}}\right)\right) \\
& =2 A_{+} e^{-\sigma_{+} / \sqrt{\varepsilon}}\left(1+\frac{Q^{\prime \prime}\left(s_{+}\right) A_{+}}{2 \sigma_{+} \sqrt{\varepsilon}} e^{-\sigma_{+} / \sqrt{\varepsilon}}+O\left(e^{-\sigma_{+} / \sqrt{\varepsilon}}\right)\right)
\end{aligned}
$$

or, equivalently,

$$
u_{1}\left(k_{0}\right)=s_{+}-2 A_{+} e^{-\sigma_{+} / \sqrt{\varepsilon}}\left(1+\frac{Q^{\prime \prime}\left(s_{+}\right) A_{+}}{2 \sigma_{+} \sqrt{\varepsilon}} e^{-\sigma_{+} / \sqrt{\varepsilon}}+O\left(e^{-\sigma_{+} / \sqrt{\varepsilon}}\right)\right)
$$

as $\varepsilon \rightarrow 0$.

To prove (3.1), we first restrict $x$ to the interval $[-1,0]$, and put

$$
u_{L}(x)=f\left(\frac{x+1}{\sqrt{\varepsilon}}\right) .
$$

From the proof of Lemma 1, it is readily seen that $u_{L}(x)$ satisfies

$$
\varepsilon u_{L}^{\prime \prime}+Q\left(u_{L}\right)=0
$$

and

$$
u_{L}(-1)=0, \quad u_{L}^{\prime}(-1)=k_{\max } .
$$

Hence, it follows from Lemma 1(a) that

$$
u_{L}^{\prime}(-1)=k_{\max }>u_{0,1}^{\prime}(-1)=k_{0} .
$$

We further claim that

$$
u_{L}(x)>u_{0,1}(x)
$$

for $x \in(-1,0]$. Assume, to the contrary, that there exists a point $x=\eta$ in the right neighborhood of $x=-1$ such that $u_{L}(\eta)=u_{0,1}(\eta), u_{L}(x)>u_{0,1}(x)$ for $x \in(-1, \eta)$ and

$$
u_{L}^{\prime}(\eta) \leq u_{0,1}^{\prime}(\eta)
$$

Using energy equations like (2.7) associated with $u_{L}(x)$ and $u_{0,1}(x)$, we have

$$
\frac{\varepsilon}{2}\left(u_{L}^{\prime}(\eta)\right)^{2}+\int_{0}^{u_{L}(\eta)} Q(s) d s=\frac{\varepsilon}{2} k_{\max }^{2}
$$

and

$$
\frac{\varepsilon}{2}\left(u_{0,1}^{\prime}(\eta)\right)^{2}+\int_{0}^{u_{0,1}(\eta)} Q(s) d s=\frac{\varepsilon}{2} k_{0}^{2}
$$


Since $k_{\max }>k_{0}, u_{L}^{\prime}(\eta)>0$, and $u_{0,1}^{\prime}(\eta)>0$, we conclude from (3.15) and (3.16) that

$$
u_{L}^{\prime}(\eta)>u_{0,1}^{\prime}(\eta)
$$

which contradicts (3.14), thus establishing (3.13). Let $s=b$ be the last point in the interval $\left(0, s_{+}\right)$such that $Q^{\prime}(b)=0$ and $Q^{\prime}(s)<0$ for $s \in\left(b, s_{+}\right]$. Correspondingly, we denote by $x_{0,1, b}$, and $x_{L, b}$ the points in the interval $[-1,0]$ such that

$$
u_{0,1}\left(x_{0,1, b}\right)=b
$$

and

$$
u_{L}\left(x_{L, b}\right)=b .
$$

From (3.15), we can derive as in (3.5)

$$
x_{L, b}+1=\sqrt{\varepsilon} \int_{s_{+}-b}^{s_{+}} \frac{d t}{\sqrt{2} \sqrt{t^{2} g(t)}} .
$$

Similarly, from (3.16), we obtain

$$
\begin{aligned}
x_{0,1, b}+1 & =\sqrt{\varepsilon} \int_{s_{+}-b}^{s_{+}} \frac{d t}{\sqrt{2} \sqrt{t^{2} g(t)-a^{2} g(a)}} \\
& =\sqrt{\varepsilon} \int_{s_{+}-b}^{s_{+}} \frac{d t}{\sqrt{2} \sqrt{t^{2} g(t)}}+O\left(\sqrt{\varepsilon} a^{2}\right) .
\end{aligned}
$$

Subtracting (3.17) from (3.18) yields

$$
d:=x_{0,1, b}-x_{L, b}=O\left(\sqrt{\varepsilon} a^{2}\right) .
$$

By the same argument, we also have

$$
x+1=\sqrt{\varepsilon} \int_{s_{+}-u_{L}(x)}^{s_{+}} \frac{d t}{\sqrt{2} \sqrt{t^{2} g(t)}}
$$

and

$$
x+1=\sqrt{\varepsilon} \int_{s_{+}-u_{0,1}(x)}^{s_{+}} \frac{d t}{\sqrt{2} \sqrt{t^{2} g(t)}}+O\left(\sqrt{\varepsilon} a^{2}\right)
$$

for $-1 \leq x \leq x_{0,1, b}$; see (3.17) and (3.18). Since $t^{2} g(t)$ is bounded away from zero for $t \in\left[s_{+}-b, s_{+}\right]$, it follows from (3.19) and (3.20) that

$$
u_{L}(x)-u_{0,1}(x)=O\left(a^{2}\right)
$$

for $-1 \leq x \leq x_{0,1, b}$.

For $x_{0,1, b} \leq x \leq 0$, the function $u_{L}(x-d)$ satisfies (3.12) and hence (3.15). Furthermore, $u_{0,1}(x)$ satisfies $(3.16)$ and

$$
u_{L}\left(x_{0,1, b}-d\right)=b=u_{0,1}\left(x_{0,1, b}\right)
$$


Since $k_{\max }>k_{0}$, we obtain

$$
u_{L}^{\prime}\left(x_{0,1, b}-d\right)>u_{0,1}^{\prime}\left(x_{0,1, b}\right) .
$$

Based on (3.22), one can use an argument similar to that for (3.13) to deduce

$$
u_{L}(x-d) \geq u_{0,1}(x) \geq b
$$

for $x_{0,1, b} \leq x \leq 0$.

Now, let $R(x)$ denote the difference between $u_{L}(x-d)$ and $u_{0,1}(x)$, i.e.,

$$
R(x):=u_{L}(x-d)-u_{0,1}(x) .
$$

From the differential equation, we have

$$
\begin{aligned}
R^{\prime \prime}(x) & =u_{L}^{\prime \prime}(x-d)-u_{0,1}^{\prime \prime}(x) \\
& =\frac{1}{\varepsilon}\left(-Q\left(u_{L}(x-d)\right)+Q\left(u_{0,1}(x)\right)\right) \geq 0 .
\end{aligned}
$$

The last inequality follows from the fact that $Q(s)$ is nonincreasing for $s \geq b$.

The convexity of $R(x)$ implies that $R^{\prime}(x) \geq R^{\prime}\left(x_{0,1, b}\right)>0$ by (3.22). Since $R\left(x_{0,1, b}\right)=0$,

$$
0 \leq R(x)<R(0)=u_{L}(0-d)-u_{0,1}(0)=O(a)
$$

for $x_{0,1, b} \leq x \leq 0$. To demonstrate the O-estimate in (3.26), we first note that $u_{0,1}(0)=$ $u_{1}\left(k_{0}\right)$ and $u_{L}(0-d)=u_{L}(0)+O\left(a^{2}\right)$. Since $u_{L}(0)=f(1 / \sqrt{\varepsilon})$, the desired estimate now follows from (2.5) and (3.11). In view of the fact that $u_{L}(x-d)-u_{L}(x)=O\left(a^{2}\right)$, we have by coupling (3.21) and (3.26)

$$
u_{L}(x)-u_{0,1}(x)=O(a)=O\left(e^{-\sigma_{+} / \sqrt{\varepsilon}}\right)
$$

for any $x \in[-1,0]$. Recall that in (3.1), the second term satisfies

$$
f\left(\frac{1-x}{\sqrt{\varepsilon}}\right)=s_{+}+O\left(e^{-\sigma_{+} / \sqrt{\varepsilon}}\right)
$$

when $x \in[-1,0]$, on account of (2.5). Therefore, (3.27) infers that (3.1) is true. When $x$ lies in $[0,1]$, the proof is similar and hence omitted. This completes the proof of $(3.1)$, and thus the theorem.

REMARK 4. If the interval $[-1,1]$ in the BVP $(1.1)-(1.2)$ is replaced by $[a, b]$, then we have instead of (3.1) and (3.2), respectively,

$$
u_{0,1}(x)=f\left(\frac{x-a}{\sqrt{\varepsilon}}\right)+f\left(\frac{b-x}{\sqrt{\varepsilon}}\right)-s_{+}+O\left(e^{-(b-a) \sigma_{+} / 2 \sqrt{\varepsilon}}\right)
$$

and

$$
u_{0,2}(x)=f\left(-\frac{x-a}{\sqrt{\varepsilon}}\right)+f\left(-\frac{b-x}{\sqrt{\varepsilon}}\right)-s_{-}+O\left(e^{-(b-a) \sigma_{-} / 2 \sqrt{\varepsilon}}\right) .
$$

For the solutions to the BVP (1.1) - (1.2) which have one internal shock layer, we have the following result. 
THEOREM 3. There exist exactly two solutions to the BVP (1.1) - (1.2), each of which has one internal shock. The first solution starts at the end point $x=-1$ with an upper arch, followed by a lower arch; while the second one starts at the end point $x=-1$ with an lower arch, followed by a upper arch. The asymptotic formulas for these two exact solutions $u_{1,1}(x)$ and $u_{1,2}(x)$ are given by

$$
\begin{aligned}
u_{1,1}(x)= & f\left(\frac{x+1}{\sqrt{\varepsilon}}\right)+f\left(-\frac{x-\bar{x}_{1}}{\sqrt{\varepsilon}}\right)+f\left(\frac{x-1}{\sqrt{\varepsilon}}\right) \\
& -\left(s_{+}+s_{-}\right)+O\left\{\exp \left(-\frac{\sigma_{+} \sigma_{-}}{\left(\sigma_{+}+\sigma_{-}\right) \sqrt{\varepsilon}}\right)\right\}
\end{aligned}
$$

and

$$
\begin{aligned}
u_{1,2}(x)=u_{1,1}(-x)= & f\left(-\frac{x+1}{\sqrt{\varepsilon}}\right)+f\left(\frac{x+\bar{x}_{1}}{\sqrt{\varepsilon}}\right)+f\left(\frac{1-x}{\sqrt{\varepsilon}}\right) \\
& -\left(s_{+}+s_{-}\right)+O\left\{\exp \left(-\frac{\sigma_{+} \sigma_{-}}{\left(\sigma_{+}+\sigma_{-}\right) \sqrt{\varepsilon}}\right)\right\}
\end{aligned}
$$

where

$$
\bar{x}_{1}=-\frac{\sigma_{+}-\sigma_{-}}{\sigma_{+}+\sigma_{-}}+\frac{2 \sqrt{\varepsilon}}{\sigma_{+}+\sigma_{-}} \log \frac{\sigma_{+} A_{+}}{\sigma_{-} A_{-}}+B \exp \left(-\frac{\sigma_{+} \sigma_{-}}{\left(\sigma_{+}+\sigma_{-}\right) \sqrt{\varepsilon}}\right)
$$

and

$$
B=\frac{Q^{\prime \prime}\left(s_{+}\right) A_{+} \sigma_{-}}{\left(\sigma_{+}+\sigma_{-}\right)^{2} \sigma_{+}}\left(\frac{\sigma_{+} A_{+}}{\sigma_{-} A_{-}}\right)^{-\frac{\sigma_{+}}{\sigma_{+}+\sigma_{-}}}+\frac{Q^{\prime \prime}\left(s_{-}\right) A_{-} \sigma_{+}}{\left(\sigma_{+}+\sigma_{-}\right)^{2} \sigma_{-}}\left(\frac{\sigma_{+} A_{+}}{\sigma_{-} A_{-}}\right)^{\frac{\sigma_{-}}{\sigma_{+}+\sigma_{-}}} .
$$

Proof. As before, we give only the proof of (3.30). Consider the function $T_{1}(k)+T_{2}(k)$ for $0<k<k_{\max }$. By Lemma 2 and $(2.27)$, we have

$$
\lim _{k \rightarrow 0} T_{1}(k)+T_{2}(k)=O(\sqrt{\varepsilon})
$$

and

$$
\lim _{k \rightarrow k_{\max }} T_{1}(k)+T_{2}(k)=\infty
$$

Hence, we conclude that there exists at least one value $k_{0} \in\left(0, k_{\max }\right)$ such that

$$
T_{1}\left(k_{0}\right)+T_{2}\left(k_{0}\right)=2 .
$$

The fact that there is only one such $k_{0}$ can be established as in Theorem 2; we shall denote by $u_{1,1}(x)$ the solution to $(1.1)-(1.2)$ with initial slope $k_{0}$ at $x=-1$. For $-k_{\max }<k<0$, there also exists a value $k_{0}$ such that (3.33) holds; its corresponding solution to (1.1) $-(1.2)$ will be denoted by $u_{1,2}(x)$. Next, we shall locate the position of $x_{1}$, the zero of $u_{1,1}(x)$. From the definition of $T_{1}\left(k_{0}\right)$ in $(2.24)$, we can show as in (3.11) that

$$
u_{1}\left(k_{0}\right)=s_{+}-2 A_{+} e^{-T_{1}\left(k_{0}\right) \sigma_{+} / 2 \sqrt{\varepsilon}}\left\{1+\frac{Q^{\prime \prime}\left(s_{+}\right) A_{+} T_{1}\left(k_{0}\right)}{4 \sigma_{+} \sqrt{\varepsilon}} e^{-T_{1}\left(k_{0}\right) \sigma_{+} / 2 \sqrt{\varepsilon}}[1+O(\sqrt{\varepsilon})]\right\} .
$$

Similarly, we also have

$$
u_{2}\left(k_{0}\right)=s_{-}+2 A_{-} e^{-T_{2}\left(k_{0}\right) \sigma_{-} / 2 \sqrt{\varepsilon}}\left\{1-\frac{Q^{\prime \prime}\left(s_{-}\right) A_{-} T_{2}\left(k_{0}\right)}{4 \sigma_{-} \sqrt{\varepsilon}} e^{-T_{2}\left(k_{0}\right) \sigma_{-} / 2 \sqrt{\varepsilon}}[1+O(\sqrt{\varepsilon})]\right\} .
$$


Equation (2.16) states

$$
-\int_{u_{2}\left(k_{0}\right)}^{0} Q(s) d s=\int_{0}^{u_{1}\left(k_{0}\right)} Q(s) d s
$$

which by Taylor's expansion gives

$$
\begin{aligned}
& \sigma_{-}^{2} A_{-}^{2} e^{-T_{2}\left(k_{0}\right) \sigma_{-} / \sqrt{\varepsilon}}\left\{1-\frac{Q^{\prime \prime}\left(s_{-}\right) A_{-} T_{2}\left(k_{0}\right)}{2 \sigma_{-} \sqrt{\varepsilon}} e^{-T_{2}\left(k_{0}\right) \sigma_{-} / 2 \sqrt{\varepsilon}}[1+O(\sqrt{\varepsilon})]\right\} \\
& =\sigma_{+}^{2} A_{+}^{2} e^{-T_{1}\left(k_{0}\right) \sigma_{+} / \sqrt{\varepsilon}}\left\{1+\frac{Q^{\prime \prime}\left(s_{+}\right) A_{+} T_{1}\left(k_{0}\right)}{2 \sigma_{+} \sqrt{\varepsilon}} e^{-T_{1}\left(k_{0}\right) \sigma_{+} / 2 \sqrt{\varepsilon}}[1+O(\sqrt{\varepsilon})]\right\} .
\end{aligned}
$$

In (3.36), we now insert $T_{1}\left(k_{0}\right)=x_{1}+1, T_{2}\left(k_{0}\right)=1-x_{1}$, and solve for $x_{1}$. This yields

$$
\begin{aligned}
x_{1}= & -\frac{\sigma_{+}-\sigma_{-}}{\sigma_{+}+\sigma_{-}}+\frac{2 \sqrt{\varepsilon}}{\sigma_{+}+\sigma_{-}} \log \frac{\sigma_{+} A_{+}}{\sigma_{-} A_{-}}+B \exp \left(-\frac{\sigma_{+} \sigma_{-}}{\left(\sigma_{+}+\sigma_{-}\right) \sqrt{\varepsilon}}\right) \\
& +O\left\{\sqrt{\varepsilon} \exp \left(-\frac{\sigma_{+} \sigma_{-}}{\left(\sigma_{+}+\sigma_{-}\right) \sqrt{\varepsilon}}\right)\right\} .
\end{aligned}
$$

Note that the sum of the first three terms in (3.37) is exactly the value $\bar{x}_{1}$ given in (3.32). On the interval $\left[-1, x_{1}\right]$, by using $(3.28)$ and

$$
f\left(\frac{x_{1}-x}{\sqrt{\varepsilon}}\right)=f\left(\frac{\bar{x}_{1}-x}{\sqrt{\varepsilon}}\right)+O\left\{\exp \left(-\frac{\sigma_{+} \sigma_{-}}{\left(\sigma_{+}+\sigma_{-}\right) \sqrt{\varepsilon}}\right)\right\}
$$

we obtain

$$
u_{1,1}(x)=f\left(\frac{x+1}{\sqrt{\varepsilon}}\right)+f\left(\frac{\bar{x}_{1}-x}{\sqrt{\varepsilon}}\right)-s_{+}+O\left\{\exp \left(-\frac{\sigma_{+} \sigma_{-}}{\left(\sigma_{+}+\sigma_{-}\right) \sqrt{\varepsilon}}\right)\right\} .
$$

Since $f\left(\frac{x-1}{\sqrt{\varepsilon}}\right)-s_{-}=O\left(e^{-\sigma_{+} \sigma_{-} /\left(\sigma_{+}+\sigma_{-}\right) \sqrt{\varepsilon}}\right)$ for $x \in\left[-1, x_{1}\right]$, (3.30) follows immediately from (3.38). Similarly, for $x \in\left[x_{1}, 1\right]$, we can use (3.29) and (3.37) to verify (3.31), thus completing the proof.

\section{APPROXIMATE SOLUTIONS WITH $N$ INTERNAL SHOCK LAYERS}

We are now ready to state and prove the general result for solutions to (1.1) - (1.2) with $n$ internal shock layers.

THEOREM 4. For any fixed nonnegative integer n, there exist exactly two solutions to the BVP (1.1) - (1.2), each having $n$ shock layers. The asymptotic formulas to these solutions $u_{n, 1}$ and $u_{n, 2}$ are given by

(i) if $n$ is odd, then

$$
\begin{aligned}
u_{n, 1}(x)= & f\left(\frac{x+1}{\sqrt{\varepsilon}}\right)+\sum_{i=1}^{n} f\left((-1)^{i} \frac{x-\bar{x}_{i}}{\sqrt{\varepsilon}}\right)+f\left(\frac{x-1}{\sqrt{\varepsilon}}\right) \\
& -\frac{n+1}{2}\left(s_{+}+s_{-}\right)+O\left\{\exp \left(-\frac{2 \sigma_{+} \sigma_{-}}{(n+1)\left(\sigma_{+}+\sigma_{-}\right) \sqrt{\varepsilon}}\right)\right\}
\end{aligned}
$$


and

$$
u_{n, 2}(x)=u_{n, 1}(-x),
$$

where

$$
\begin{aligned}
\bar{x}_{i}= & -1+\left[\frac{i+1}{2}\right] \frac{4}{n+1} \frac{\sigma_{-}}{\sigma_{+}+\sigma_{-}}+\left[\frac{i}{2}\right] \frac{4}{n+1} \frac{\sigma_{+}}{\sigma_{+}+\sigma_{-}} \\
& +\left(\left[\frac{i+1}{2}\right]-\left[\frac{i}{2}\right]\right) \frac{2 \sqrt{\varepsilon}}{\sigma_{+}+\sigma_{-}} \log \frac{\sigma_{+} A_{+}}{\sigma_{-} A_{-}} \\
& +\left(\left[\frac{i+1}{2}\right]-\left[\frac{i}{2}\right]\right) B(n) \exp \left(-\frac{2 \sigma_{+} \sigma_{-}}{(n+1)\left(\sigma_{+}+\sigma_{-}\right) \sqrt{\varepsilon}}\right),
\end{aligned}
$$

and

$$
\begin{aligned}
B(n)= & \frac{2 Q^{\prime \prime}\left(s_{+}\right) A_{+} \sigma_{-}}{(n+1)\left(\sigma_{+}+\sigma_{-}\right)^{2} \sigma_{+}}\left(\frac{\sigma_{+} A_{+}}{\sigma_{-} A_{-}}\right)^{-\frac{\sigma_{+}}{\sigma_{+}+\sigma_{-}}} \\
& +\frac{2 Q^{\prime \prime}\left(s_{-}\right) A_{-} \sigma_{+}}{(n+1)\left(\sigma_{+}+\sigma_{-}\right)^{2} \sigma_{-}}\left(\frac{\sigma_{+} A_{+}}{\sigma_{-} A_{-}}\right)^{\frac{\sigma_{-}}{\sigma_{+}+\sigma_{-}}}
\end{aligned}
$$

(ii) if $n$ is even, then

$$
\begin{aligned}
u_{n, 1}(x)= & f\left(\frac{x+1}{\sqrt{\varepsilon}}\right)+\sum_{i=1}^{n} f\left((-1)^{i} \frac{x-\bar{x}_{i}}{\sqrt{\varepsilon}}\right)+f\left(-\frac{x-1}{\sqrt{\varepsilon}}\right) \\
& -\left(\frac{n}{2}+1\right) s_{+}-\frac{n}{2} s_{-}+O\left\{\exp \left(-\frac{2 \sigma_{+} \sigma_{-}}{(n+1)\left(\sigma_{+}+\sigma_{-}\right) \sqrt{\varepsilon}}\right)\right\}
\end{aligned}
$$

where $\bar{x}_{i}$ is still given by (4.2), and

$$
\begin{aligned}
u_{n, 2}(x)= & f\left(-\frac{x+1}{\sqrt{\varepsilon}}\right)+\sum_{i=1}^{n} f\left((-1)^{i+1} \frac{x-\bar{x}_{i}}{\sqrt{\varepsilon}}\right)+f\left(\frac{x-1}{\sqrt{\varepsilon}}\right) \\
& -\frac{n}{2} s_{+}-\left(\frac{n}{2}+1\right) s_{-}+O\left\{\exp \left(-\frac{2 \sigma_{+} \sigma_{-}}{(n+1)\left(\sigma_{+}+\sigma_{-}\right) \sqrt{\varepsilon}}\right)\right\},
\end{aligned}
$$

where

$$
\begin{aligned}
\bar{x}_{i}= & -1+\left[\frac{i}{2}\right] \frac{4}{n+1} \frac{\sigma_{-}}{\sigma_{+}+\sigma_{-}}+\left[\frac{i+1}{2}\right] \frac{4}{n+1} \frac{\sigma_{+}}{\sigma_{+}+\sigma_{-}} \\
& +\left(\left[\frac{i}{2}\right]-\left[\frac{i+1}{2}\right]\right) \frac{2 \sqrt{\varepsilon}}{\sigma_{+}+\sigma_{-}} \log \frac{\sigma_{+} A_{+}}{\sigma_{-} A_{-}} \\
& -\left(\left[\frac{i+1}{2}\right]-\left[\frac{i}{2}\right]\right) B(n) \exp \left(-\frac{2 \sigma_{+} \sigma_{-}}{(n+1)\left(\sigma_{+}+\sigma_{-}\right) \sqrt{\varepsilon}}\right) .
\end{aligned}
$$

Proof. (i) Consider the function

$$
\frac{n+1}{2} T_{1}(k)+\frac{n+1}{2} T_{2}(k) .
$$

In a manner similar to that in the proof of Theorem 3 , one can show that there exists a $k_{0}$ such that

$$
\frac{n+1}{2} T_{1}\left(k_{0}\right)+\frac{n+1}{2} T_{2}\left(k_{0}\right)=2
$$


and

$$
\frac{n+1}{2} T_{1}\left(-k_{0}\right)+\frac{n+1}{2} T_{2}\left(-k_{0}\right)=2,
$$

which, in turn, means that when $n$ is odd, there exist exact two solutions to the BVP (1.1) - (1.2) with $n$ shock layers.

By making use of (4.6), one can solve $T_{1}\left(k_{0}\right)$ and $T_{2}\left(k_{0}\right)$ in (3.36). This yields

$$
\begin{aligned}
T_{1}\left(k_{0}\right)= & \frac{4}{n+1} \frac{\sigma_{-}}{\sigma_{+}+\sigma_{-}}+\frac{2 \sqrt{\varepsilon}}{\sigma_{+}+\sigma_{-}} \log \frac{\sigma_{+} A_{+}}{\sigma_{-} A_{-}} \\
& +B(n) \exp \left(-\frac{2 \sigma_{+} \sigma_{-}}{(n+1)\left(\sigma_{+}+\sigma_{-}\right) \sqrt{\varepsilon}}\right)+O\left\{\sqrt{\varepsilon} \exp \left(-\frac{2 \sigma_{+} \sigma_{-}}{(n+1)\left(\sigma_{+}+\sigma_{-}\right) \sqrt{\varepsilon}}\right)\right\}
\end{aligned}
$$

and

$$
\begin{aligned}
T_{2}\left(k_{0}\right)= & \frac{4}{n+1} \frac{\sigma_{+}}{\sigma_{+}+\sigma_{-}}-\frac{2 \sqrt{\varepsilon}}{\sigma_{+}+\sigma_{-}} \log \frac{\sigma_{+} A_{+}}{\sigma_{-} A_{-}} \\
& -B(n) \exp \left(-\frac{2 \sigma_{+} \sigma_{-}}{(n+1)\left(\sigma_{+}+\sigma_{-}\right) \sqrt{\varepsilon}}\right)+O\left\{\sqrt{\varepsilon} \exp \left(-\frac{2 \sigma_{+} \sigma_{-}}{(n+1)\left(\sigma_{+}+\sigma_{-}\right) \sqrt{\varepsilon}}\right)\right\} .
\end{aligned}
$$

It is easily verified that

$$
x_{i}=-1+\left[\frac{i+1}{2}\right] T_{1}\left(k_{0}\right)+\left[\frac{i}{2}\right] T_{2}\left(k_{0}\right), \quad i=1,2, \cdots, n .
$$

Hence, as in (3.37), one obtains

$$
\begin{aligned}
x_{i}= & -1+\left[\frac{i+1}{2}\right] \frac{4}{n+1} \frac{\sigma_{-}}{\sigma_{+}+\sigma_{-}}+\left[\frac{i}{2}\right] \frac{4}{n+1} \frac{\sigma_{+}}{\sigma_{+}+\sigma_{-}} \\
& +\left(\left[\frac{i+1}{2}\right]-\left[\frac{i}{2}\right]\right) \frac{2 \sqrt{\varepsilon}}{\sigma_{+}+\sigma_{-}} \log \frac{\sigma_{+} A_{+}}{\sigma_{-} A_{-}} \\
& +\left(\left[\frac{i+1}{2}\right]-\left[\frac{i}{2}\right]\right) B(n) \exp \left(-\frac{2 \sigma_{+} \sigma_{-}}{(n+1)\left(\sigma_{+}+\sigma_{-}\right) \sqrt{\varepsilon}}\right) \\
& +O\left\{\sqrt{\varepsilon} \exp \left(-\frac{2 \sigma_{+} \sigma_{-}}{(n+1)\left(\sigma_{+}+\sigma_{-}\right) \sqrt{\varepsilon}}\right)\right\} .
\end{aligned}
$$

Note that $x_{i}$ approximates $\bar{x}_{i}$ in (4.2) within the $O$-term in the last equation.

For the proof of (4.1), we split the interval $[-1,1]$ into subintervals $\left[-1, x_{1}\right],\left[x_{i}, x_{i+1}\right], i=$ $1,2, \cdots, n-1$, and $\left[x_{n}, 1\right]$. The validity of $(4.1)$ is established in each of these intervals; for example, in $\left[-1, x_{1}\right]$ we have, as in Theorems 2 and 3 ,

$$
u_{n, 1}(x)=f\left(\frac{x+1}{\sqrt{\varepsilon}}\right)+f\left(-\frac{x-\bar{x}_{1}}{\sqrt{\varepsilon}}\right)-s_{+}+O\left\{\exp \left(-\frac{2 \sigma_{+} \sigma_{-}}{(n+1)\left(\sigma_{+}+\sigma_{-}\right) \sqrt{\varepsilon}}\right)\right\} .
$$

Since

$$
\begin{aligned}
s_{+}+\sum_{i=2}^{n} f\left((-1)^{i} \frac{x-\bar{x}_{i}}{\sqrt{\varepsilon}}\right)+f\left(\frac{x-1}{\sqrt{\varepsilon}}\right) & -\frac{n+1}{2}\left(s_{+}+s_{-}\right) \\
& =O\left\{\exp \left(-\frac{2 \sigma_{+} \sigma_{-}}{(n+1)\left(\sigma_{+}+\sigma_{-}\right) \sqrt{\varepsilon}}\right)\right\}
\end{aligned}
$$

for $x \in\left[-1, x_{1}\right]$, we conclude that (4.1) holds in this interval.

The proofs of (4.3) and (4.4) are entirely similar and hence omitted. This completes the proof of the theorem. 


\section{BOUNDARY CONDITIONS (1.3)}

In this section we are concerned with the boundary conditions in (1.3). As we shall see, in this case the solutions to (1.1) \& (1.3) do not have shocks at endpoints.

We again assume that $Q(s)$ satisfies the condition in Lemma 4, i.e.,

$$
s\left(\frac{Q(s)}{s}\right)^{\prime}<0
$$

for $s \in\left[s_{-}, s_{+}\right]$and $s \neq 0$. As in Theorem 1, the following result can be established.

THEOREM 5. Let $n_{1}(\varepsilon)$ denote the number of shocks that a solution to the BVP $(1.1) \&(1.3)$ can have, and let $n_{2}(\varepsilon)$ denote the number of solutions to (1.1) \& (1.3). If the condition in (5.1) holds, then we have

$$
1 \leq n_{1}(\varepsilon) \leq N(\varepsilon)
$$

and

$$
n_{2}(\varepsilon)=2 N(\varepsilon)
$$

where $N(\varepsilon)$ is as defined in (2.36).

It should be noted that condition (5.1) is not required in establishing the asymptotic formulas for the exact solutions to BVP (1.1) \& (1.3) when $\varepsilon$ is small. Indeed, we have

THEOREM 6. For any fixed nonnegative integer $n$, there exist exactly two solutions to (1.1) \& (1.3), each having $n$ shock layers. The asymptotic formulas to these solutions $u_{n, 1}$ and $u_{n, 2}$ are given by

(i) if $n$ is odd then

$$
\begin{aligned}
u_{n, 1}(x)= & \sum_{i=1}^{n} f\left((-1)^{i+1} \frac{x-\bar{x}_{i}}{\sqrt{\varepsilon}}\right) \\
& -\frac{n-1}{2}\left(s_{+}+s_{-}\right)+O\left\{\exp \left(-\frac{2 \sigma_{+} \sigma_{-}}{n\left(\sigma_{+}+\sigma_{-}\right) \sqrt{\varepsilon}}\right)\right\}
\end{aligned}
$$

and

$$
u_{n, 2}(x)=u_{n, 1}(-x)
$$

where

$$
\begin{aligned}
\bar{x}_{i}= & -1+\frac{2}{n} \frac{\sigma_{+}}{\sigma_{+}+\sigma_{-}}-\frac{\sqrt{\varepsilon}}{\sigma_{+}+\sigma_{-}} \log \frac{\sigma_{+} A_{+}}{\sigma_{-} A_{-}} \\
& +\left[\frac{i}{2}\right]\left(\frac{4}{n} \frac{\sigma_{-}}{\sigma_{+}+\sigma_{-}}+\frac{2 \sqrt{\varepsilon}}{\sigma_{+}+\sigma_{-}} \log \frac{\sigma_{+} A_{+}}{\sigma_{-} A_{-}}\right) \\
& +\left[\frac{i-1}{2}\right]\left(\frac{4}{n} \frac{\sigma_{+}}{\sigma_{+}+\sigma_{-}}-\frac{2 \sqrt{\varepsilon}}{\sigma_{+}+\sigma_{-}} \log \frac{\sigma_{+} A_{+}}{\sigma_{-} A_{-}}\right) \\
& +\left(-\frac{1}{2}+\left[\frac{i}{2}\right]-\left[\frac{i-1}{2}\right]\right) B(n-1) \exp \left(-\frac{2 \sigma_{+} \sigma_{-}}{n\left(\sigma_{+}+\sigma_{-}\right) \sqrt{\varepsilon}}\right)
\end{aligned}
$$


and $B(n)$ is defined as in Theorem 4;

(ii) if $n$ is even then

$$
u_{n, 1}(x)=\sum_{i=1}^{n} f\left((-1)^{i+1} \frac{x-\bar{x}_{i}}{\sqrt{\varepsilon}}\right)-\frac{n}{2} s_{+}-\frac{n-2}{2} s_{-}+O\left\{\exp \left(-\frac{2 \sigma_{+} \sigma_{-}}{n\left(\sigma_{+}+\sigma_{-}\right) \sqrt{\varepsilon}}\right)\right\}
$$

where $\bar{x}_{i}$ is as given in (5.3), and

$$
u_{n, 2}(x)=\sum_{i=1}^{n} f\left((-1)^{i} \frac{x-x_{i}}{\sqrt{\varepsilon}}\right)-\frac{n-2}{2} s_{+}-\frac{n}{2} s_{-}+O\left\{\exp \left(-\frac{2 \sigma_{+} \sigma_{-}}{n\left(\sigma_{+}+\sigma_{-}\right) \sqrt{\varepsilon}}\right)\right\}
$$

where

$$
\begin{aligned}
\bar{x}_{i}= & -1+\frac{2}{n} \frac{\sigma_{-}}{\sigma_{+}+\sigma_{-}}+\frac{\sqrt{\varepsilon}}{\sigma_{+}+\sigma_{-}} \log \frac{\sigma_{+} A_{+}}{\sigma_{-} A_{-}} \\
& +\left[\frac{i}{2}\right]\left(\frac{4}{n} \frac{\sigma_{+}}{\sigma_{+}+\sigma_{-}}-\frac{2 \sqrt{\varepsilon}}{\sigma_{+}+\sigma_{-}} \log \frac{\sigma_{+} A_{+}}{\sigma_{-} A_{-}}\right) \\
& +\left[\frac{i-1}{2}\right]\left(\frac{4}{n} \frac{\sigma_{-}}{\sigma_{+}+\sigma_{-}}+\frac{2 \sqrt{\varepsilon}}{\sigma_{+}+\sigma_{-}} \log \frac{\sigma_{+} A_{+}}{\sigma_{-} A_{-}}\right) \\
& -\left(-\frac{1}{2}+\left[\frac{i}{2}\right]-\left[\frac{i-1}{2}\right]\right) B(n-1) \exp \left(-\frac{2 \sigma_{+} \sigma_{-}}{n\left(\sigma_{+}+\sigma_{-}\right) \sqrt{\varepsilon}}\right) .
\end{aligned}
$$

Proof. (i) Let $k_{0}$ denote the slope of $u_{n, 1}$ at the point $x=x_{1}$, the first zero of $u_{n, 1}$ from the left. As before, we denote by $T_{1}\left(k_{0}\right)$ and $T_{2}\left(k_{0}\right)$ the length of the upper and lower arch, respectively. Then we have

$$
\frac{n}{2} T_{1}\left(k_{0}\right)+\frac{n}{2} T_{2}\left(k_{0}\right)=2 .
$$

Using (5.6), one can solve $T_{1}$ and $T_{2}$ in (3.36). This gives

$$
\begin{aligned}
T_{1}\left(k_{0}\right)= & \frac{4}{n} \frac{\sigma_{-}}{\sigma_{+}+\sigma_{-}}+\frac{2 \sqrt{\varepsilon}}{\sigma_{+}+\sigma_{-}} \log \frac{\sigma_{+} A_{+}}{\sigma_{-} A_{-}} \\
& +B(n-1) \exp \left(-\frac{2 \sigma_{+} \sigma_{-}}{n\left(\sigma_{+}+\sigma_{-}\right) \sqrt{\varepsilon}}\right)+O\left\{\sqrt{\varepsilon} \exp \left(-\frac{2 \sigma_{+} \sigma_{-}}{n\left(\sigma_{+}+\sigma_{-}\right) \sqrt{\varepsilon}}\right)\right\}
\end{aligned}
$$

and

$$
\begin{aligned}
T_{2}(k)= & \frac{4}{n} \frac{\sigma_{+}}{\sigma_{+}+\sigma_{-}}-\frac{2 \sqrt{\varepsilon}}{\sigma_{+}+\sigma_{-}} \log \frac{\sigma_{+} A_{+}}{\sigma_{-} A_{-}} \\
& -B(n-1) \exp \left(-\frac{2 \sigma_{+} \sigma_{-}}{n\left(\sigma_{+}+\sigma_{-}\right) \sqrt{\varepsilon}}\right)+O\left\{\sqrt{\varepsilon} \exp \left(-\frac{2 \sigma_{+} \sigma_{-}}{n\left(\sigma_{+}+\sigma_{-}\right) \sqrt{\varepsilon}}\right)\right\} .
\end{aligned}
$$

Since

$$
x_{i}=-1+\frac{T_{2}\left(k_{0}\right)}{2}+\left[\frac{i}{2}\right] T_{1}\left(k_{0}\right)+\left[\frac{i-1}{2}\right] T_{2}\left(k_{0}\right),
$$


it follows that

$$
\begin{aligned}
x_{i}= & -1+\frac{2}{n} \frac{\sigma_{+}}{\sigma_{+}+\sigma_{-}}-\frac{\sqrt{\varepsilon}}{\sigma_{+}+\sigma_{-}} \log \frac{\sigma_{+} A_{+}}{\sigma_{-} A_{-}} \\
+ & {\left[\frac{i}{2}\right]\left(\frac{4}{n} \frac{\sigma_{-}}{\sigma_{+}+\sigma_{-}}+\frac{2 \sqrt{\varepsilon}}{\sigma_{+}+\sigma_{-}} \log \frac{\sigma_{+} A_{+}}{\sigma_{-} A_{-}}\right) } \\
+ & {\left[\frac{i-1}{2}\right]\left(\frac{4}{n} \frac{\sigma_{+}}{\sigma_{+}+\sigma_{-}}-\frac{2 \sqrt{\varepsilon}}{\sigma_{+}+\sigma_{-}} \log \frac{\sigma_{+} A_{+}}{\sigma_{-} A_{-}}\right) } \\
+ & \left(-\frac{1}{2}+\left[\frac{i}{2}\right]-\left[\frac{i-1}{2}\right]\right) B(n-1) \exp \left(-\frac{2 \sigma_{+} \sigma_{-}}{n\left(\sigma_{+}+\sigma_{-}\right) \sqrt{\varepsilon}}\right) \\
+ & O\left\{\sqrt{\varepsilon} \exp \left(-\frac{2 \sigma_{+} \sigma_{-}}{n\left(\sigma_{+}+\sigma_{-}\right) \sqrt{\varepsilon}}\right)\right\} .
\end{aligned}
$$

Neglecting the $O$-term in $x_{i}$, we obtain $\bar{x}_{i}$ given in (5.3).

In a manner analogous to that in the proof of Theorem 5 , we can also deduce

$$
u_{n, 1}(x)=\sum_{i=1}^{n} f\left((-1)^{i+1} \frac{x-x_{i}}{\sqrt{\varepsilon}}\right)-\frac{n-1}{2}\left(s_{+}+s_{-}\right)+O\left\{\exp \left(-\frac{2 \sigma_{+} \sigma_{-}}{n\left(\sigma_{+}+\sigma_{-}\right) \sqrt{\varepsilon}}\right)\right\}
$$

and

$$
u_{n, 2}(x)=u_{n, 1}(-x) .
$$

(ii) The argument for this case is similar.

\section{TYPICAL EXAMPLES}

In this section we present two particular examples to illustrate our main results.

(a) When $Q(u)=2 u-2 u^{3}$, it is easy to calculate that $s_{-}=-1, s_{+}=1, Q^{\prime}( \pm 1)=-4$, $\sigma_{ \pm}=2$

$$
\int_{-1}^{1} Q(s) d s=0
$$

and

$$
f(x)=\tanh (x) .
$$

Applying Theorem 4, we have the following result.

COROLLARY 3. For any fixed nonnegative integer $n$, there exist exactly two solutions to the BVP (1.1) - (1.2), each having $n$ shock layers. The asymptotic formulas to these solutions $u_{n, 1}$ and $u_{n, 2}$ are given by

(i) if $n$ is odd, then

$$
\begin{aligned}
u_{n, 1}(x, \varepsilon)= & \tanh \left(\frac{x+1}{\sqrt{\varepsilon}}\right)+\sum_{i=1}^{n} \tanh \left((-1)^{i} \frac{x-\bar{x}_{i}}{\sqrt{\varepsilon}}\right)+\tanh \left(\frac{x-1}{\sqrt{\varepsilon}}\right) \\
& +O\left(e^{-2 /(n+1) \sqrt{\varepsilon}}\right)
\end{aligned}
$$


and

$$
u_{n, 2}(x, \varepsilon)=u_{n, 1}(-x, \varepsilon)
$$

where $\bar{x}_{i}=-1+\frac{2 i}{n+1}$

(ii) if $n$ is even, then

$$
\begin{aligned}
u_{n, 1}(x, \varepsilon)= & \tanh \left(\frac{x+1}{\sqrt{\varepsilon}}\right)+\sum_{i=1}^{n} \tanh \left((-1)^{i} \frac{x-\bar{x}_{i}}{\sqrt{\varepsilon}}\right)+\tanh \left(-\frac{x-1}{\sqrt{\varepsilon}}\right) \\
& -1+O\left(e^{-2 /(n+1) \sqrt{\varepsilon}}\right)
\end{aligned}
$$

and

$$
u_{n, 2}(x, \varepsilon)=-u_{n, 1}(x, \varepsilon)
$$

where $\bar{x}_{i}$ is as given in (i).

(b) When $Q(u)=\sin \pi u$, we have $Q^{\prime}( \pm 1)=-\pi, \sigma_{ \pm}=\sqrt{\pi}, s_{-}=-1, s_{+}=1$,

$$
\int_{-1}^{1} Q(s) d s=0
$$

and

$$
f(x)=\frac{4}{\pi} \tan ^{-1}\left(\frac{-1+e^{\sqrt{\pi} x}}{1+e^{\sqrt{\pi} x}}\right) .
$$

Although $Q(u)$ has infinitely many zeros on the $u$-axis, Theorem 4 still applies and we have the following corollary.

COROLLARY 4. For any fixed nonnegative integer $n$, there exist exactly two solutions to the BVP (1.1) - (1.2), each having $n$ shock layers. Let $f(x)$ be given in (6.4). Then, the asymptotic formulas to these solutions $u_{n, 1}$ and $u_{n, 2}$ are given by

(i) if $n$ is odd, then

$$
\begin{aligned}
u_{n, 1}(x, \varepsilon)= & f\left(\frac{x+1}{\sqrt{\varepsilon}}\right)+\sum_{i=1}^{n} f\left((-1)^{i} \frac{x-\bar{x}_{i}}{\sqrt{\varepsilon}}\right)+f\left(\frac{x-1}{\sqrt{\varepsilon}}\right) \\
& +O\left(e^{-\sqrt{\pi} /(n+1) \sqrt{\varepsilon}}\right)
\end{aligned}
$$

and

$$
u_{n, 2}(x, \varepsilon)=u_{n, 1}(-x, \varepsilon)
$$

where $\bar{x}_{i}=-1+\frac{2 i}{n+1}$

(ii) if $n$ is even, then

$$
\begin{aligned}
u_{n, 1}(x, \varepsilon)= & f\left(\frac{x+1}{\sqrt{\varepsilon}}\right)+\sum_{i=1}^{n} f\left((-1)^{i} \frac{x-\bar{x}_{i}}{\sqrt{\varepsilon}}\right)+f\left(-\frac{x-1}{\sqrt{\varepsilon}}\right) \\
& -1+O\left(e^{-\sqrt{\pi} /(n+1) \sqrt{\varepsilon}}\right)
\end{aligned}
$$


and

$$
u_{n, 2}(x, \varepsilon)=-u_{n, 1}(x, \varepsilon)
$$

where $\bar{x}_{i}$ is as given in (i).

REMARK 5. In the above two corollaries, if we change the boundary conditions in (1.2) to those in (1.3), then corresponding asymptotic formulas can be obtained by dropping the terms $\tanh \left(\frac{x+1}{\sqrt{\varepsilon}}\right)$ and $\tanh \left(\frac{x-1}{\sqrt{\varepsilon}}\right)$ in $(6.2), \tanh \left(\frac{x+1}{\sqrt{\varepsilon}}\right)$ and $\tanh \left(-\frac{x-1}{\sqrt{\varepsilon}}\right)$ in $(6.3), f\left(\frac{x+1}{\sqrt{\varepsilon}}\right)$ and $f\left(\frac{x-1}{\sqrt{\varepsilon}}\right)$ in $(6.5)$, and $f\left(\frac{x+1}{\sqrt{\varepsilon}}\right)$ and $f\left(-\frac{x-1}{\sqrt{\varepsilon}}\right)$ in $(6.6)$; see Theorem 6 .

\section{DISCUSSION}

In this final section, we discuss two separate issues. The first issue concerns a set of mixed boundary conditions studied in [8] by Ward, and the second one deals with the situation where the nonlinear term $Q(u)$ in (1.1) is replaced by $-Q(u)$. Throughout the section, we shall continue to assume that $Q(u)$ satisfies the conditions imposed in Sec. 2.

We first consider the mixed boundary conditions

$$
\sqrt{\varepsilon} u^{\prime}(1)+k_{r}\left(u(1)-s_{+}\right)=0
$$

and

$$
\sqrt{\varepsilon} u^{\prime}(-1)-k_{l}\left(u(-1)-s_{-}\right)=0,
$$

where both $k_{r}$ and $k_{l}$ are nonnegative numbers. We claim that solutions of equation (1.1) under these boundary conditions have properties very similar to those established in the previous sections. For simplicity of illustration, we restrict ourselves to the case in which there is only one shock in $(-1,1)$, and let $x_{1}$ denote the location of the shock, i.e., $u\left(x_{1}\right)=0$. Furthermore, let $k_{0}$ denote the derivative of $u(x)$ at $x_{1}$. In what follows, we shall derive asymptotic formulas for $x_{1}$ and $k_{0}$. To proceed, we bear in mind that on one hand, $u(x)$ is a solution of the boundary-value problem (1.1), (7.1) \& (7.2), and on the other hand, it satisfies the initial value $u\left(x_{1}\right)=0$ and $u^{\prime}\left(x_{1}\right)=k_{0}$. As before, we search values for $k_{0}$ in the interval $\left|k_{0}\right|<k_{\max }$.

We consider only the case $k_{0} \in\left(0, k_{\max }\right)$; the other case $k \in\left(-k_{\max }, 0\right)$ can be handled in a similar manner. Since $k_{0} \in\left(0, k_{\max }\right)$, from the proof of Lemma $1(\mathrm{c})$ we know that $u(x)$ is period and intersects the $x$-axis infinitely many times. Furthermore, $s_{-}<u(x)<s_{+}$for $x \in(-\infty, \infty)$. Using this fact, we also have from (7.1) and (7.2)

$$
u^{\prime}( \pm 1) \geq 0
$$

Since we have assumed that there is only one shock in $[-1,1]$, using equation $(2.7)$ one can readily see that $u(x)$ is not decreasing there.

Let $z_{1}>1, z_{2}<-1$ be the two nearest points to $x_{1}$ satisfying $u^{\prime}\left(z_{1}\right)=u^{\prime}\left(z_{2}\right)=0$. Then, we have $u\left(z_{1}\right)=u_{1}\left(k_{0}\right), u\left(z_{2}\right)=u_{2}\left(k_{0}\right), z_{1}-x_{1}=\frac{1}{2} T_{1}\left(k_{0}\right), x_{1}-z_{2}=\frac{1}{2} T_{2}\left(k_{0}\right)$. Here 
$u_{1}\left(k_{0}\right), u_{2}\left(k_{0}\right), T_{1}\left(k_{0}\right)$ and $T_{2}\left(k_{0}\right)$ are defined as before. Similar to (3.36), one can derive

$$
\begin{aligned}
& \sigma_{-}^{2} A_{-}^{2} e^{-T_{2}\left(k_{0}\right) \sigma_{-} / \sqrt{\varepsilon}}\left\{1-\frac{Q^{\prime \prime}\left(s_{-}\right) A_{-} T_{2}\left(k_{0}\right)}{2 \sigma_{-} \sqrt{\varepsilon}} e^{-T_{2}\left(k_{0}\right) \sigma_{-} / 2 \sqrt{\varepsilon}}[1+O(\sqrt{\varepsilon})]\right\} \\
& =\sigma_{+}^{2} A_{+}^{2} e^{-T_{1}\left(k_{0}\right) \sigma_{+} / \sqrt{\varepsilon}}\left\{1+\frac{Q^{\prime \prime}\left(s_{+}\right) A_{+} T_{1}\left(k_{0}\right)}{2 \sigma_{+} \sqrt{\varepsilon}} e^{-T_{1}\left(k_{0}\right) \sigma_{+} / 2 \sqrt{\varepsilon}}[1+O(\sqrt{\varepsilon})]\right\} .
\end{aligned}
$$

Integrating (2.23) from $x_{1}$ to 1 , we obtain

$$
1-x_{1}=\sqrt{\varepsilon} \int_{s_{+}-u(1)}^{s_{+}} \frac{d t}{\sqrt{2} \sqrt{t^{2} g(t)-a^{2} g(a)}}
$$

c.f. (3.5). The integral on the right-hand side can be evaluated as in (3.10), and we have

$$
1-x_{1} \sim \sqrt{\varepsilon}\left[\frac{1}{\sigma_{+}}\left(\cosh ^{-1} \frac{s_{+}}{a}-\cosh ^{-1} \frac{s_{+}-u(1)}{a}\right)+c_{+}\right],
$$

where $a=s_{+}-u_{1}\left(k_{0}\right)$. By the same argument, from (2.24) and the equation following (3.10) we get

$$
\begin{aligned}
T_{1}\left(k_{0}\right) & =\sqrt{2 \varepsilon} \int_{a}^{s_{+}} \frac{d t}{\sqrt{t^{2} g(t)-a^{2} g(a)}} \\
& \sim 2 \sqrt{\varepsilon}\left(\frac{1}{\sigma_{+}} \cosh ^{-1} \frac{s_{+}}{a}+c_{+}\right) .
\end{aligned}
$$

Coupling (7.4) and (7.5) gives

$$
1-x_{1} \sim \frac{T_{1}\left(k_{0}\right)}{2}-\frac{\sqrt{\varepsilon}}{\sigma_{+}} \cosh ^{-1} \frac{s_{+}-u(1)}{a},
$$

from which it follows by (3.34) that

$$
u(1) \sim s_{+}-A_{+} e^{-\sigma_{+}\left(1-x_{1}\right) / \sqrt{\varepsilon}}-A_{+} e^{-\sigma_{+}\left(T_{1}\left(k_{0}\right)-\left(1-x_{1}\right)\right) / \sqrt{\varepsilon}} .
$$

Using (2.23), we can also deduce

$$
u^{\prime}(1) \sim \frac{A_{+} \sigma_{+}}{\sqrt{\varepsilon}} e^{-\sigma_{+}\left(1-x_{1}\right) / \sqrt{\varepsilon}}-\frac{A_{+} \sigma_{+}}{\sqrt{\varepsilon}} e^{-\sigma_{+}\left(T_{1}\left(k_{0}\right)-\left(1-x_{1}\right)\right) / \sqrt{\varepsilon}} .
$$

Substituting (7.7) and (7.8) into (7.1) yields

$$
A_{+}^{2} \sigma_{+}^{2} \frac{\sigma_{+}-k_{r}}{\sigma_{+}+k_{r}} e^{-2 \sigma_{+}\left(1-x_{1}\right) / \sqrt{\varepsilon}} \sim A_{+}^{2} \sigma_{+}^{2} e^{-T_{1}\left(k_{0}\right) \sigma_{+} / \sqrt{\varepsilon}} .
$$

Similarly, at the endpoint $x=-1$ we have

$$
A_{-}^{2} \sigma_{-}^{2} \frac{\sigma_{-}-k_{l}}{\sigma_{-}+k_{l}} e^{-2 \sigma_{-}\left(1+x_{1}\right) / \sqrt{\varepsilon}} \sim A_{-}^{2} \sigma_{-}^{2} e^{-T_{2}\left(k_{0}\right) \sigma_{-} / \sqrt{\varepsilon}} .
$$


By a combination of (7.3), (7.9) and (7.10), we obtain

$$
\begin{aligned}
x_{1}= & \frac{\sigma_{+}-\sigma_{-}}{\sigma_{+}+\sigma_{-}} \\
& -\frac{\sqrt{\varepsilon}}{2\left(\sigma_{+}+\sigma_{-}\right)}\left[2 \log \left(\frac{A_{+} \sigma_{+}}{A_{-} \sigma_{-}}\right)+\log \left(\frac{\gamma_{+}}{\gamma_{-}}\right)\right] \\
& -D \exp \left(-\frac{2 \sigma_{+} \sigma_{-}}{\left(\sigma_{+}+\sigma_{-}\right) \sqrt{\varepsilon}}\right)+O\left\{\sqrt{\varepsilon} \exp \left(-\frac{2 \sigma_{+} \sigma_{-}}{\left(\sigma_{+}+\sigma_{-}\right) \sqrt{\varepsilon}}\right)\right\},
\end{aligned}
$$

where

$$
\gamma_{+}=\frac{\sigma_{+}-k_{r}}{\sigma_{+}+k_{r}}, \quad \gamma_{-}=\frac{\sigma_{-}-k_{l}}{\sigma_{-}+k_{l}}
$$

and

$$
D=\frac{Q^{\prime \prime}\left(s_{+}\right) A_{+} \sigma_{-}}{\left(\sigma_{+}+\sigma_{-}\right)^{2} \sigma_{+}}\left(\frac{A_{+}^{2} \sigma_{+}^{2} \gamma_{+}}{A_{-}^{2} \sigma_{-}^{2} \gamma_{-}}\right)^{-\frac{\sigma_{+}}{2\left(\sigma_{+}+\sigma_{-}\right)}}+\frac{Q^{\prime \prime}\left(s_{-}\right) A_{-} \sigma_{+}}{\left(\sigma_{+}+\sigma_{-}\right)^{2} \sigma_{-}}\left(\frac{A_{+}^{2} \sigma_{+}^{2} \gamma_{+}}{A_{-}^{2} \sigma_{-}^{2} \gamma_{-}}\right)^{\frac{\sigma-}{2\left(\sigma_{+}+\sigma_{-}\right)}}
$$

The first two terms of $x_{1}$ in (7.11) agree exactly with the formula given in Ward [8]. Inserting (7.11) into (7.9), and using (3.34) and the fact

$$
\frac{\varepsilon}{2} k_{0}^{2}=\int_{0}^{u_{1}\left(k_{0}\right)} Q(s) d s,
$$

we also get the asymptotic formula

$$
k_{0} \sim k_{\max }-\frac{\sigma_{+}^{2} A_{+}^{2}}{\varepsilon k_{\max }} \exp \left\{-\frac{4 \sigma_{+} \sigma_{-}}{\sqrt{\varepsilon}\left(\sigma_{+}+\sigma_{-}\right)}\right\} .
$$

When $\sigma_{+}>k_{r}$ and $\sigma_{-}>k_{l}, \log \gamma_{+}$and $\log \gamma_{+}$are well defined. With the values of $x_{1}$ and $k_{0}$ given by (7.11) and (7.12), we conclude that the solution $u(x)$ to our initial value problem exists, and has the asymptotic formula

$$
u(x)=f\left(\frac{x-x_{1}}{\sqrt{\varepsilon}}\right)+O\left\{\exp \left(-\frac{2 \sigma_{+} \sigma_{-}}{\left(\sigma_{+}+\sigma_{-}\right) \sqrt{\varepsilon}}\right)\right\} .
$$

Next, we consider the problem when $Q(u)$ in (1.1) is replaced by $-Q(u)$. To our surprise, solutions now exhibit new phenomena. For instance, let us take $Q(u)=-2 u\left(1-u^{2}\right)$. In this case, we still have condition (1.4), i.e.,

$$
\int_{s_{-}}^{s_{+}} Q(s)=0
$$

but the pattern of solutions is completely changed. All solutions of (1.1) exhibit spike layers instead of shock layers, and the values of each solution are close to 0, except those near the spike regions; see a typical solution in Figure 4 below 


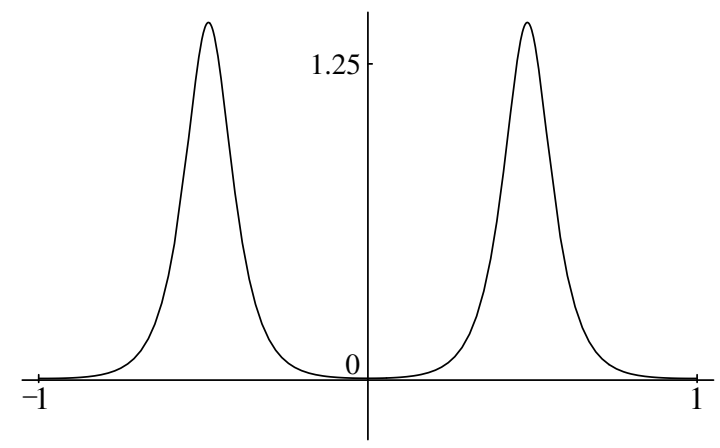

Figure 4. $u^{\prime}(-1)=u^{\prime}(1)=0$

When condition (1.4) does not hold, we find that like the above case, every solution still exhibits only spikes. For example, if we take $Q(u)=u(u+1)(2-u)$, we obtain a solution which is close to $u=-1$, except at three spikes; see Figure 5 .

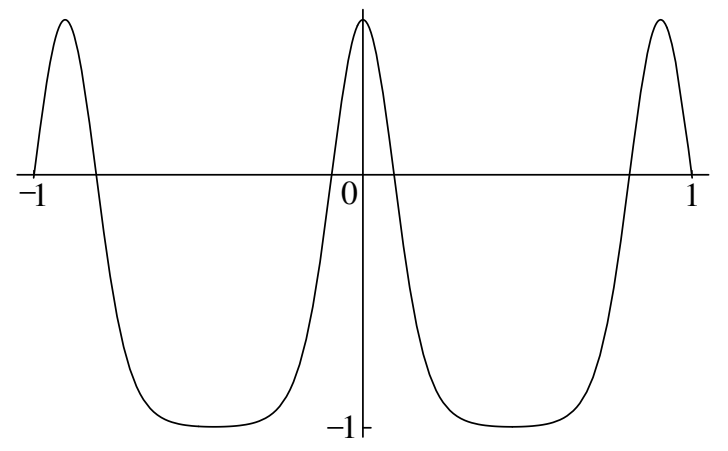

Figure 5. $u(-1)=u(1)=0$

If we allow $Q(u)$ to have the shape shown in Figure 6, i.e., $Q(u)$ vanishes at $s_{1}, s_{2}, 0, s_{3}, s_{4}$ and nowhere else, and satisfies

$$
\int_{s_{2}}^{s_{3}} Q(s)=0
$$




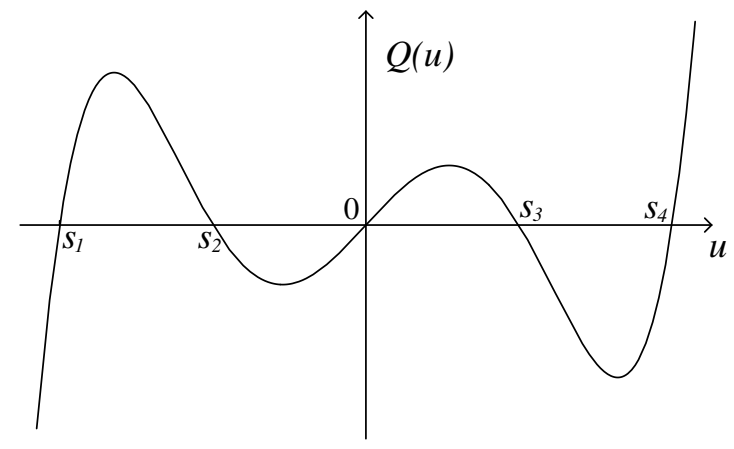

Figure 6. Graph of $Q(u)$

then the problem can have solutions with both spike-type and shock-type internal layers. For instance, if we take $Q(u)=u\left(u^{2}-1\right)\left(u^{2}-4\right)$, then a typical solution is depicted in Figure 7.

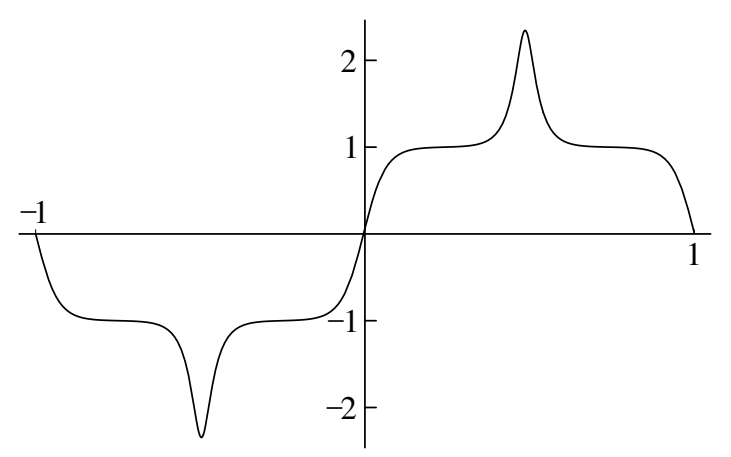

Figure 7. Graph of $u(x)$

Using the shooting method that we have introduced in the previous paper [6] and used again in the present paper, we can prove the results that we have observed above with mathematical rigor.

\section{REFERENCES}

1. G. F. Carrier and C. E. Pearson, Ordinary Differential Equations, Blaisdell Pub. Co., Waltham, MA, 1968. (Reprinted in SIAM's Classics in Applied Mathematics series, vol. 6, SIAM, Philadelphia, 1991.)

2. W. Kath, C. Knessl and B. Matkowsky, A variational approach to singularly perturbed boundary value problems, Studies in Appl. Math., 77 (1987), 61-88. 
3. C. G. Lange, On spurious solutions of singular perturbation problems, Studies in Appl. Math., 68 (1983), 227-257.

4. A. D. MacGillivray, A method for incorporating transcendentally small terms into the method of matched asymptotic expansions, Studies in Appl. Math., 99 (1997), 285-310.

5. R. E. O'Malley, Jr., Phase-plane solutions to some singular perturbation problems, J. Math. Anal. Appl., 54 (1976), 449-466.

6. C. H. Ou and R. Wong, On a two-point boundary value problem with spurious solutions, Studies in Appl. Math. (to appear).

7. L. G. Reyna and M. J. Ward, Resolving weak internal layer interactions for the Ginzburg-Landau equation, European J. Appl. Math., 5 (1994), 495-523.

8. M. J. Ward, Eliminating indeterminacy in singularly perturbed boundary value problems with translation invariant potentials, Studies in Appl. Math., 87 (1992), 95-134. 